\title{
The role of regulated necrosis in endocrine diseases
}

\section{Wulf Tonnus (D), Alexia Belavgeni(D), Felix Beuschlein (1), Graeme Eisenhofer, Martin Fassnacht $(\mathbb{D}$, Matthias Kroiss $(\mathbb{D}$, , Nils P. Krone, Martin Reincke (1), Stefan R. Bornstein and Andreas Linkermann (D)}

Abstract | The death of endocrine cells is involved in type 1 diabetes mellitus, autoimmunity, adrenopause and hypogonadotropism. Insights from research on basic cell death have revealed that most pathophysiologically important cell death is necrotic in nature, whereas regular metabolism is maintained by apoptosis programmes. Necrosis is defined as cell death by plasma membrane rupture, which allows the release of damage-associated molecular patterns that trigger an immune response referred to as necroinflammation. Regulated necrosis comes in different forms, such as necroptosis, pyroptosis and ferroptosis. In this Perspective, with a focus on the endocrine environment, we introduce these cell death pathways and discuss the specific consequences of regulated necrosis. Given that clinical trials of necrostatins for the treatment of autoimmune conditions have already been initiated, we highlight the therapeutic potential of such novel therapeutic approaches that, in our opinion, should be tested in endocrine disorders in the future.

Endocrine glands control the human body's metabolism, vital functions, mental and physical adaptation to stress, and the capacity to reproduce. Regulated cell necrosis and cell proliferation define endocrine tissue development and homeostasis, as well as its capacity to adapt to stress and to prevent tumour formation. The term regulated cell death was used mostly synonymously with apoptosis until 15 years ago. However, our understanding of necrosis as a regulated process has dramatically expanded since then. In the past 15 years, we have begun to understand the details of necroptosis, pyroptosis and ferroptosis ${ }^{1}$. Necrosis not only causes organ dysfunction but also results in an inflammatory response driven by damage-associated molecular patterns (DAMPs), which is referred to as necroinflammation ${ }^{2,3}$.

Studies have shown that the three pathways of regulated necrosis, namely necroptosis, pyroptosis and ferroptosis, can be therapeutically targeted ${ }^{4-6}$. Accumulating data suggest that necroinflammation is of central relevance in the pathophysiology of endocrine disorders ${ }^{7,8}$, including septic adrenal dysfunction, autoimmune diseases such as type 1 diabetes mellitus (T1DM) ${ }^{9}$ and endocrine malignancies. Both the prevention of cell death by rescuing cells that otherwise succumb to necrosis (as is the case in T1DM) ${ }^{9}$ and the induction of necrosis in endocrine cancers (such as with adrenocortical carcinomas) ${ }^{10}$ might lead to therapeutic benefits. This Perspective therefore aims to summarize the existing data on the newly characterized cell death pathways in endocrine disorders while focusing on unanswered questions. We also discuss the need to revisit the literature that was published before the identification of necroptosis, pyroptosis and ferroptosis and reinterpret it in the light of the current understanding to highlight the possibility of therapeutic interventions.

\section{The types of regulated cell death Apoptosis: well defined and well tolerated by the immune system. Apoptosis (FIC. 1 a) is the default cell death pathway for homeostasis and cellular turnover during physiological metabolism ${ }^{11,12}$. The caspase-controlled}

system results in the typical morphological features such as rapid shrinking of the cell, nuclear condensation, DNA fragmentation, exposure of phosphatidylserine and a process known as membrane blebbing ${ }^{11,12}$. Phosphatidylserine exposure functions as an 'eat me' signal to macrophages ${ }^{13-15}$. Importantly, the plasma membrane remains intact in apoptotically dying cells, a mechanism that prevents the release of intracellular content to the interstitial and/or extracellular space. Therefore, apoptosis is immunologically silent. The detection of apoptosis has been misinterpreted for decades by the TdT-mediated dUTP-biotin nick end-labelling (TUNEL) method (BOX 1).

Mechanistically, extrinsic apoptosis is mediated by death receptors such as tumour necrosis factor receptor 1 (TNFR1) or the FAS receptor (also known as CD95 ${ }^{16}$. To kill a cell through a TNFR1 signal, upon binding of the ligand TNF, the TNFR1 signalling complex dissociates from the plasma membrane. Within the cytosol, the FAS-associated death domain protein (FADD)-bound initiator caspase 8 is recruited to the complex and interacts with receptor-interacting protein kinase 1 $(\mathrm{RIPK} 1)^{17,18}$. In the case of TNFR1, however, the default signal to activate the transcription factor NF- $\kappa \mathrm{B}$ is maintained through the recruitment of kinases (IKKe and TBK1) through linear polyubiquitin chains on RIPK1 (REF. ${ }^{19}$ ) and does not result in cell death. Mechanistically, TBK1 phosphorylates RIPK1 and thereby inhibits its autophosphorylation-induced activation, which is required for cell death. However, upon activation of caspase 8, RIPK1 activity is prevented by proteolytic cleavage of RIPK1 by caspase $8\left(\mathrm{REF}^{20}\right)$. The proteolytically active caspase 8 also cleaves the effectors caspase 3, caspase 6 and caspase $7\left(\mathrm{REF}^{21}{ }^{21}\right.$, which mediate the apoptotic cellular phenotype. As caspase 8 also controls the activation of the pyroptosis pathway ${ }^{22}$, it must be interpreted as the master regulator of death receptor-mediated cell death. The control of extrinsic apoptosis and necroptosis (which is discussed in the next section) by post-translational modification is reviewed in more detail elsewhere ${ }^{23-25}$.

By contrast, loss of the mitochondrial outer membrane potential and the recruitment of BAX and BAK to the outer 
a Apoptosis

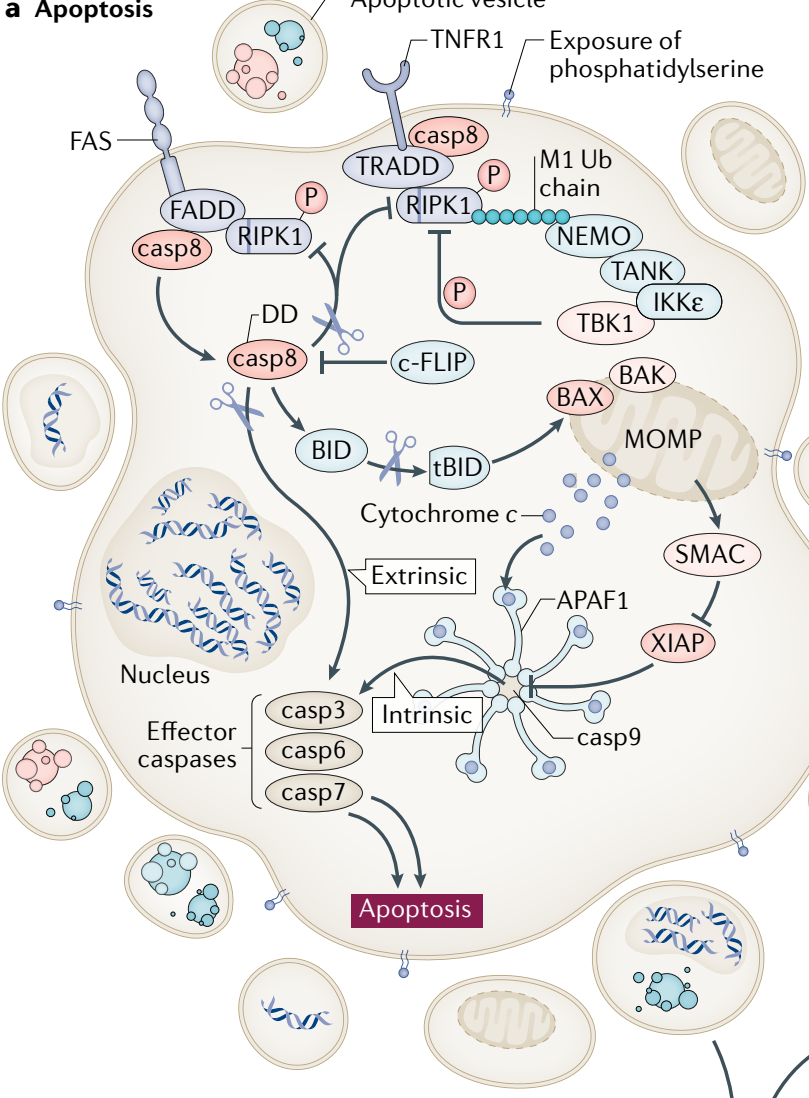

\section{c Pyroptosis}

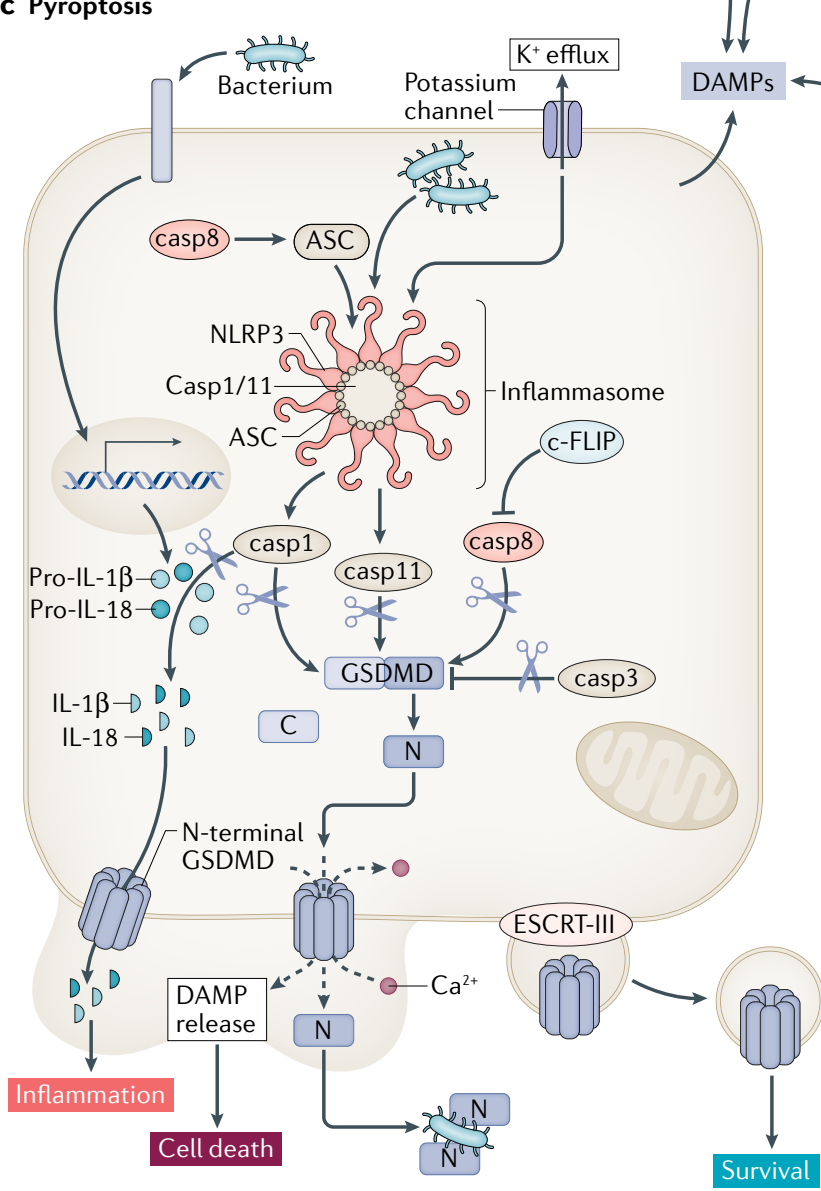

b Necroptosis

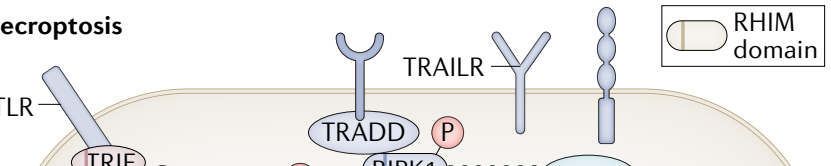

Viral DNA and/or RNA
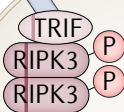

(P) RIPK1 0000000 NEMO TANK

TAK1 P) 8 (TIPK3
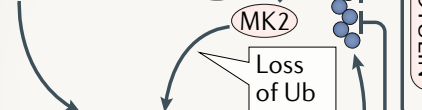

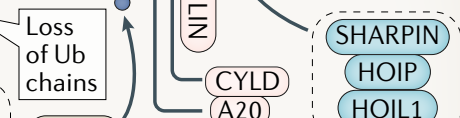

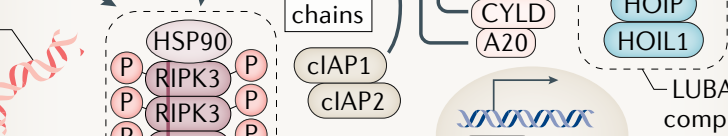

(RIPK3) P

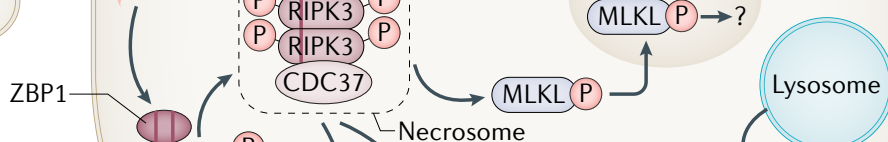
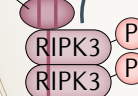

- Lecrosome
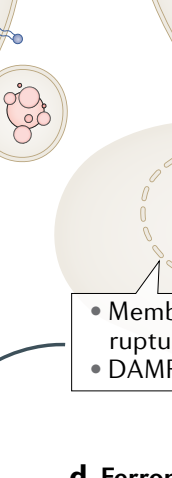

Glutamate 9
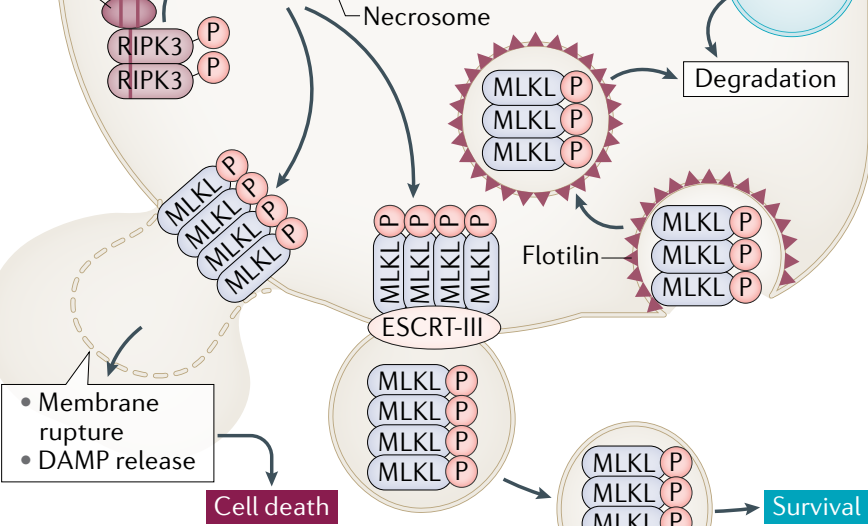

ESCRT-III

$M L K L P$
$M L K L(P$
$M L K L P$

MLKL(P) MLKL(P)

$\stackrel{M L K L(P)}{M L K L(P)} \rightarrow$ Survival

MLKLP 
4 Fig. $1 \mid$ Regulated cell death pathways at a glance. Apoptosis represents a non-inflammatory pathway that is mediated by caspases (part a). Two distinct signalling pathways of apoptosis, extrinsic and intrinsic apoptosis, have been characterized. While apoptosis depends on the activation of caspases, necroptosis is mediated by kinases (part b). Dependent on its RHIM domain, RIPK3 forms an amyloidlike structure referred to as the necrosome, the central signalling platform of necroptosis. Therein, RIPK3 phosphorylates the pseudokinase MLKL. By unknown mechanisms, the phosphorylated form of MLKL (pMLKL) triggers plasma membrane rupture, a process that was demonstrated to be counteracted by the membrane repair ESCRT-III complex. In contrast to apoptosis and necroptosis, inflammasomes are involved in the initiation of cell death by pyroptosis (part c). The activation of caspases $(1,4,11)$ in inflammasomes results in the cleavage of pro-IL-1 $\beta$, pro-IL-18 and gasdermin D (GSDMD). Finally, and without any clear connection to apoptosis, necroptosis and pyroptosis, ferroptosis is a failsafe rather than a typical cell death pathway (part d). In cellular homeostasis, $\mathrm{H}_{2} \mathrm{O}_{2}$ concentrations of iron-catalysed and Fenton reactions are limited by diverse cellular anti-redox systems. Glutathione peroxidase 4 (GPX4) prevents lipid peroxidation that otherwise leads to plasma membrane rupture by unknown mechanisms. By contrast, the oxidoreductase FSP1 (also known as AIFM2) prevents lipid peroxidation upon myristoylation-dependent recruitment to the plasma membrane in a GSHindependent manner. casp, caspase; DAMP, damage-associated molecular pattern; DD, death domain; DED, death effector domain; ER, endoplasmic reticulum; FA, fatty acid; PL-OH, phospholipid alcohol; $\mathrm{PL}-\mathrm{OOH}$, phospholipid hydroperoxide; RTA, radical-trapping antioxidant.

mitochondrial membrane result in the release of mitochondrial content into the cytosol $^{26}$. For instance, cytochrome c assembles with the cytosolic proteins APAF1 and caspase 9 , which results in the formation of the apoptosome, a large heteroheptameric structure that activates the effectors caspase 3, caspase 6 and caspase 7 to execute intrinsic apoptosis ${ }^{27-29}$.

\section{Necroptosis: MLKL-mediated necrosis to defend viruses. Necroptosis is thought to} be evolutionarily conserved as a pathway to defend against viral infection. However, the necroptosis machinery might also function in endocrine disease even in the absence of infection. During evolution, viruses developed mechanisms to evade their host's defence and the immune systems ${ }^{30,31}$. Upon the viral inhibition of caspase 8, RIPK1 and RIPK3 are no longer inhibited by the caspases' proteolytic activity ${ }^{30,31}$. The failure of caspase 8 to cleave RIPK1, which can be a consequence of human mutations, results in autoimmunity ${ }^{32,33}$. In such situations and upon the genetic loss of caspase 8, RIPK3 oligomerizes $^{34,35}$ through its RIP homotypic interaction motif (RHIM) domain to form a large amyloid-like platform ${ }^{36,37}$, which is referred to as the necrosome.

In addition to RIPK1 and RIPK3, there are only two other human encoded proteins that contain a similar RHIM domain (FIG. 1 b). One such protein, Z-DNA-binding protein 1 (ZBP1; also known as DAI), recognizes intracellular oligonucleotides ${ }^{38-43}$, thereby merging another detection strategy to the necroptosis machinery as these oligonucleotides typically appear during viral infection. Along similar lines, the TIR domain-containing adapter-inducing interferon- $\beta$ (TRIF) contains a RHIM domain and links the necroptosis machinery to bacterial host recognition ${ }^{44-46}$. In all of these cases, mixed lineage kinase domain-like (MLKL) represents the downstream effector of necroptosis ${ }^{5,47}$ following phosphorylation by the RIPK3 kinase domain ${ }^{48-50}$. It is unclear how pMLKL actually causes the loss of plasma membrane integrity, but it is clear that the endosomal sorting complexes required for the transport (ESCRT-III) complex ${ }^{15,51,52}$ antagonize necroptosis to a certain extent, most likely as a means of membrane repair. The necroptosis-driven response to single-stranded RNA viruses includes the host defence against influenza A viruses ${ }^{53}$ and SARS-CoV-2 (REF. ${ }^{54}$ ). Serum levels of RIPK3 have been demonstrated to be upregulated in patients with COVID-19 $\left(\mathrm{REF}^{55}{ }^{5}\right.$ ). Therefore, necroptosis might also represent a mechanism underlying the virally induced destruction of pancreatic $\beta$-cells ${ }^{56}$.

Although the upstream modulators of ZBP1 and TRIF have been characterized, death receptor-mediated necroptosis is the modulator that has been best characterized. As mentioned earlier in this Perspective, the membrane-bound TNF receptor signalling complex becomes untethered upon the loss of post-translational modification motifs on RIPK1, such as polyubiquitin chains. Such chains can be assembled in a different way, for example, by K48 linkages that target proteins in proteasomal degradation. However, K63 ubiquitin linkages or linear linkages (also known as M1 linkages) on RIPK1 are assembled by different E3-ligase complexes. The cellular inhibitor of apoptosis protein 1 (cIAP1) and inhibitor of apoptosis protein 2 (cIAP2) are involved in RIPK1 K63 polyubiquitination ${ }^{57,58}$. Likewise, the linear ubiquitin chain assembly complex (LUBAC), which consists of the proteins
SHARPIN, HOIP and HOIL1, generates linear polyubiquitin chains ${ }^{59-61}$. The polyubiquitin chains are disassembled by the deubiquitinases (DUBs) referred to as A20 (REFS ${ }^{62,63}$ ) and CYLD ${ }^{20,64-67}$ in the case of K63 linkages and as OTULIN ${ }^{68-70}$ in the case of linear chains. It is this subtle equilibrium of post-translational modification that determines if the kinases IKKe and TBK1 are recruited into the complex and prevent cell death signalling either by apoptosis ${ }^{71}$ or by necroptosis ${ }^{19}$. In addition, the sensitivity to necroptosis is further regulated on different phosphorylation sites on RIPK1 (REFS $\left.{ }^{23,72,73}\right)$ by the proteins MK2 (REFS ${ }^{74,75}$ ) and TAK1 (REF. ${ }^{76}$ ).

\section{Pyroptosis: gasdermin-mediated necrosis to amplify inflammasome} action. Inflammasomes are intracellular supramolecular complexes, the proteolytic activity of which is mediated by caspase 1 and caspase 11 in mice (caspase 4 in humans $)^{77,78}$. Three important cytosolic proteins contain a cleavage site for those caspases and are therefore activated by inflammasomes: pro-IL- $1 \beta$ and pro-IL-18 are cleaved by caspase 1 , gasdermin $\mathrm{D}$ (GSDMD) can be cleaved by caspase 11 $\left(\mathrm{REFS}^{79-81}\right)$. However, the mature cytokines IL- $1 \beta$ and IL-18 do not contain a sorting motif and thereby cannot be actively secreted by exocytosis. Despite being a matter of scientific controversy, it is clear that pro-inflammatory cytokines can reach interstitial space through the activation of GSDMD following its proteolytic cleavage by caspase 11 . A 28 -fold singlering pore forms from the $\mathrm{N}$-terminal

\section{Box 1 | Confusion caused by TUNEL- positive staining}

TdT-mediated dUTP-biotin nick end-labelling (TUNEL) is a method that detects DNA double-strand breaks ${ }^{223}$. To this day and to the best of our knowledge, every cell death pathway described so far is associated with TUNEL positivity. However, TUNEL detection kits were sold as 'apoptosis detection kits', which confused researchers for a long time. Therefore, it is important to now revisit the existing data on TUNEL positivity, which might in fact reveal that cells died by necroptosis, pyroptosis or ferroptosis. Additional methods to detect cleaved caspase 3 in tissues, along with the typical apoptotic morphology (shrinking, nuclear condensation and a complex process of untethering the plasma membrane from the cytoskeleton, referred to as blebbing) and sensitivity to inhibition by an appropriate caspase inhibitor, are now required for TUNEL-positive cell death to be interpreted conclusively as apoptosis ${ }^{1}$. 


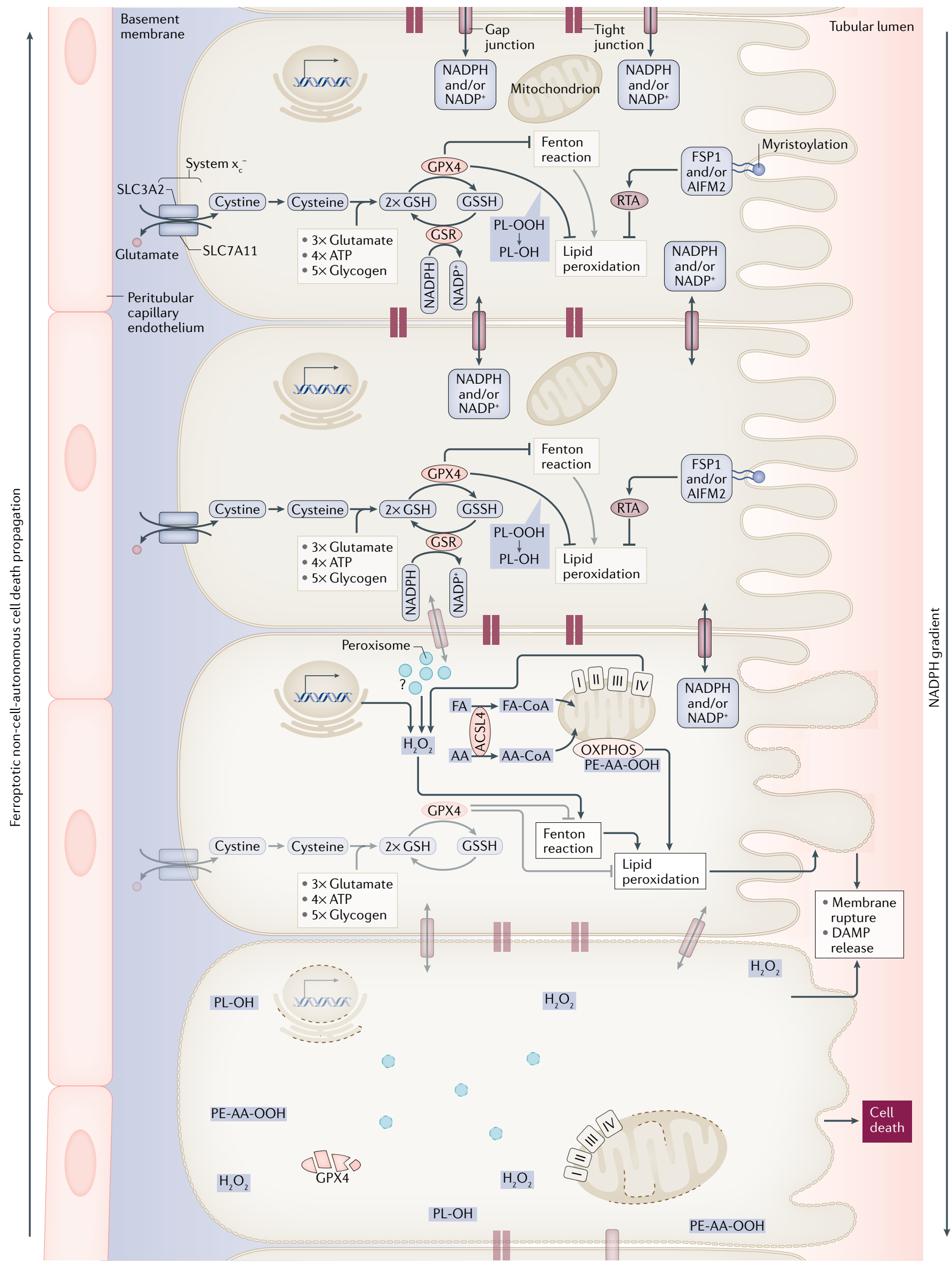


4 Fig. 2 | Synchronized regulated necrosis. In most cases, it is currently unclear how a necrotic zone of dead cells propagates during an event of sepsis or ischaemia. Based on time-lapse videos obtained from isolated perfused renal tubules that underwent ferroptosis, the concept of synchronized regulated necrosis emerged ${ }^{96}$. Although this phenomenon has not been observed in humans, necrotic tubules in the urine of patients and experimental intravital microscopy videos suggest that cell death occurs in a wave of death ${ }^{97,222}$. Given the connections between cells in a functional syncytium, such as a renal tubule, the adrenal gland or the myocardium, it is tempting to speculate that the intracellular redox capacity (NADPH concentration) diffuses through intercellular junctions. If the redox capacity decreases in a dying cell, an NADPH gradient forms, which leaves the closest neighbour at high risk of undergoing ferroptosis. At least from heart attacks and insults of acute kidney injury, it is now clear that the bulk of the necrotic area that occurs during myocardial infarction or tubular necrosis originates from cells that underwent ferroptosis. We speculate that similar mechanisms might occur in the adrenal gland during sepsis or during resuscitation in intensive care units and potentially in other endocrine organs. AA, arachidonic acid; DAMP, damage-associated molecular pattern; FA, fatty acid; GPX4; glutathione peroxidase 4; GSH, glutathione; GSR, glutathione-disulfide reductase; GSSG, glutathione disulfide; HMGCR, 3-hydroxy-3-methylglutaryl-CoA reductase; $\mathrm{PL}-\mathrm{OH}$, phospholipid alcohol; PL-OOH, phospholipid hydroperoxide; RTA, radical-trapping antioxidant.

fragments, as demonstrated in detail for gasdermin A3 (REF. ${ }^{82}$ ), another member of the GSDM family, to execute necrotic cell death by pyroptosis (FIG. 1c). Importantly, GSDMD-deficient mice are protected from lipopolysaccharide-mediated shock. The role of pyroptosis in infectious diseases has been clearly established. However, in the past few years, it has become clear that gasdermins are controlled by caspase $8\left(\operatorname{REFS}^{22,83}\right)$, which was also demonstrated to function upstream of inflammasome activation ${ }^{84-87}$. Mechanistically, the death effector domain (DED) of caspase 8, at least in cell culture, was demonstrated to bind to ASC $^{22}$ but confirming data in mice are lacking to this day.

Other members of the GSDM family might exert similar pore-forming functions. It has been noted that gasdermin $\mathrm{E}$ (GSDME) can be activated by caspase 3 during apoptosis ${ }^{88,89}$. Interestingly, GSDMD also contains a caspase 3 cleavage site ${ }^{90}$, which functions to inactivate the molecule and preclude pore formation by the GSDMD N-terminal fragment ${ }^{88,89,91}$. If the ESCRT-III complex inefficiently counteracts the formation of pores by actively releasing exosomes that contain GSDMD N-terminal fragments ${ }^{92}$, a plasma membrane rupture is triggered, thus defining the process of pyroptosis $^{79-81}$. It will be interesting to see which other proteases might potentially activate members, other than GSDMD and GSDME, that do not carry a caspase cleavage site. In addition, some evidence suggests that caspase 8 might be involved in GSDMD cleavage independently of inflammasomes ${ }^{93-95}$. It will be interesting to unravel the non-inflammasome-mediated roles of gasdermins.

Ferroptosis: ancient iron-dependent necrosis by lipid peroxidation. Ferroptosis (FIG. 1d) is unlike apoptosis, necroptosis and pyroptosis in many ways. First, it is not induced by classic signalling, such as through a death receptor or a DNA sensor. Second, it is entirely unclear how the plasma membrane might rupture in ferroptosis. Finally, cell death by ferroptosis occurs in a non-cell-autonomous manner referred to as synchronized regulated necrosis. A model of cell death propagation, best studied in renal tubules ${ }^{96}$, is presented in FIG. 2. Besides kidney tubules, synchronized regulated necrosis has been reported in cell culture $^{97,98}$ and zebrafish ${ }^{99}$. A calcium signal precedes membrane rupture minutes before the cells undergo necrosis ${ }^{98}$. However, it is beyond the scope of this Perspective to list the detailed considerations of ferroptosis execution and we refer the interested reader to excellent articles on this topic ${ }^{100,101}$; it should be mentioned that it remains unclear what initiates the spread of ferroptotic cell death $^{102}$.

Ferroptosis is a necrotic-type cell death resulting from the dysfunction of molecules that, under healthy conditions, prevent lipid peroxidation ${ }^{101}$. Such redox systems become proportionally more important relative to the number of Fenton reactions that need to be controlled in given cells. The sensitivity to ferroptosis therefore varies between cell types, but our current understanding is that hormone-producing cells and cancer cells are particularly sensitive to death by lipid peroxidation ${ }^{7}$.

The most redundant and best-studied system to suppress lipid peroxidation in mammals depends on glutathione peroxidase $4(\mathrm{GPX} 4)^{103-106}$. GPX4 metabolizes glutathione (GSH) to decrease the intracellular $\mathrm{H}_{2} \mathrm{O}_{2}$ concentration. Sufficient GSH is critical for GPX4 function and is provided by GSH synthase or the mevalonate pathway that requires cysteine, imported into the cell as cystine (the oxidized dimer form of cysteine) through the Cyc-Glu antiporter system $\mathrm{x}_{\mathrm{c}}^{-}$ $\left(\right.$ REF. $\left.^{107}\right)$.

In 2020, the protein apoptosis-inducing factor mitochondria associated 2 (AIFM2) was renamed as ferroptosis suppressor protein 1 (FSP1) by two groups that discovered the oxidoreductase activity of FSP1 to effectively suppress ferroptosis in a GSH-independent manner ${ }^{108,109}$. The recruitment of FSP1 to the plasma membrane via its myristylation binding motifs seems to be required for effective ferroptosis prevention ${ }^{108,109}$.

In addition, prominin 2 was identified as another negative regulator of ferroptosis ${ }^{110}$. This pentaspanin protein is known to be implicated in the regulation of lipid dynamics and was demonstrated to sort ferritin into multivesicular bodies, the outer membrane of which might fuse with the plasma membrane and thereby release ferritin-containing vesicles from the cell ${ }^{110}$. This iron export might desensitize cells against ferroptosis, but it is currently unclear how the microenvironment might be affected by these mechanisms. The microenvironmental changes are of particular interest in light of ferroptosis as an event of synchronized regulated necrosis (FIG. 2).

Haploid cell screens have helped to identify that acyl-coenzyme A synthetase long-chain isoform 4 (ACSL4) is critical to sensitize cells to RSL3-induced ferroptosis ${ }^{11-113}$. A genetic deficiency of ACSL4 in specific organs is considered to be a knockout for ferroptosis. For the endocrine focus of this Perspective, however, it is equally important to mention that the tissue-specific ablation of ACSL 4 can be dispensable for normal steroidogenesis ${ }^{114}$.

In addition, $\mathrm{P} 450$ oxidoreductase, an endoplasmic reticulum (ER)-associated enzyme that carries disease-causing mutations in congenital adrenal hyperplasia and Antley-Bixler syndrome ${ }^{115}$, has been found to promote ferroptosis ${ }^{116,117}$. However, this function seems to be independent of its interaction with P450 enzymes required for steroidogenesis but is instead dependent on its function to generate $\mathrm{H}_{2} \mathrm{O}_{2}$. Along similar lines, CYB5R1, another ER-associated oxidoreductase, has been found to synergistically modulate ferroptosis sensitivity in cases of decreased P450 oxidoreductase activity ${ }^{117}$.

The induction of ferroptosis can be achieved in several different ways. The inhibition of system $\mathrm{x}_{c}^{-}$(by erastin) is referred to as a class 1 ferroptosis inducer $(\mathrm{FIN})^{107}$. Class 2 FINs, such as RSL3, directly target the enzymatically active pocket of 
GPX4, whereas class 3 FINs (such as FIN56) induce GPX4 degradation ${ }^{118}$. For FINO2, which is also referred to as a class $4 \mathrm{FIN}$, the mechanism of action is less clear ${ }^{119}$. Several cancers, including adrenocortical carcinomas, might be particularly sensitive to the induction of ferroptosis ${ }^{10,106,120-123}$.

\section{Interconnection between cell death}

pathways. During viral infection, necroptosis seems to be a backup cell death pathway for those virally infected cells that express caspase inhibitors ${ }^{48}$. Mechanistically, several viral proteins inhibit the proteolytic function of caspase 8 , which therefore no longer functions to activate caspase 3 , caspase 6 , caspase 7 and apoptosis ${ }^{30}$. The same proteolytic activity of caspase 8 is required for the inactivation of RIPK1 $\left(\mathrm{REF}^{20}\right)$, which in turn becomes active upon the viral inhibition of caspase 8 to pursue necroptosis ${ }^{48}$. This function requires a heterodimer complex of caspase 8 with the long form of cellular FLICE-inhibitory protein ${ }^{35}$. Impressively, along similar lines, RIPK3-deficiency rescues the lethal phenotype of caspase 8 -deficient mice ${ }^{34,35}$. Furthermore, caspase 8 might directly recruit ASC through its death effector domain ${ }^{22}$ and thereby regulate inflammasome activation and pyroptosis $^{22,83}$. In addition, it is under debate whether caspase 8 , in some circumstances, is capable of directly activating GSDMD ${ }^{93-95}$. At the level of gasdermins, another crosstalk between apoptosis and pyroptosis exists. Active caspase 3 proteolytically inhibits pyroptosis by cleavage of GSDMD at a different cleavage site ${ }^{89}$. It was further demonstrated that cellular FLICE-inhibitory protein directly inhibits caspase 8-mediated GSDMD cleavage and pyroptosis ${ }^{124}$. These examples substantiate the crosslinks between apoptosis, necroptosis and pyroptosis, most prominently regulated by caspase 8 $\left(\right.$ REF. ${ }^{125}$ ). The interconnection between cell death pathways was best demonstrated by mutations of RIPK1 that prevent caspase cleavage; such mutations result in autoinflammatory diseases ${ }^{32}$.

As a common downstream mechanism, the ESCRT-III complex prevents the loss of membrane integrity by releasing pMLKL $^{51,52}$ or GSDMD N-terminal-containing microvesicles ${ }^{22}$ from the cell surface, thereby regulating both necroptosis and pyroptosis sensitivity.

In contrast to the complex apoptosisnecroptosis-pyroptosis system, ferroptosis (with respect to what has been demonstrated to date) does not appear to be mechanistically connected in any way. Given the importance of ferroptosis, this seems to be unexpected. Indeed, the only common features of all necrosis pathways, including ferroptosis, are the loss of redox capacity during very late stage cell death ${ }^{18}$, plasma membrane rupture, and the release of DAMPs and, as introduced here for the first time, particularly in hormone-producing cells, the damage-induced release of endocrine factors (DIRE).

DAMPs and DIRE. The evolutionary conservation of more than one pathway of regulated necrosis can be explained by the differences in the immunogenicity of necroptosis, pyroptosis and ferroptosis. The requirement for delicately balancing

\section{Box 2 | DAMPs and DIRE - the consequences of necrosis of endocrine cells}

\section{Consequence one}

A dead cell does not function as a living cell. This simple fact directly affects the production of endocrine factors and the physiological role of the endocrine gland.

\section{Consequence two}

The necrotic debris following plasma membrane rupture influences the surrounding tissue. The phagocytosis of necrotic debris, for example by LC3-associated phagocytosis ${ }^{127}$, might prevent the inflammation that would otherwise follow. Such novel epitopes, released from necrotic cells, are collectively referred to as damage-associated molecular patterns (DAMPs) ${ }^{2}$ (FIG. 3). In the exceptional case of necrosis of hormone-producing cells, such as thyrotoxicosis, radioiodine treatment, surgical manipulation, infarction of hormone-producing tumours and necrotic death might be associated with hormone release. This hormone release is an endocrine cell-specific phenomenon that we will refer to as the damage-induced release of endocrine factors (DIRE). DIRE can cause specific systemic consequences mediated by hormone signalling. We consider it possible that DIRE might partially account for distant organ effects during critical illness. These effects might be triggered by the release of adrenaline, noradrenaline, tetraiodothyronine and tri-iodothyronine, and potentially other hormones. Finally, in contrast to DAMPs, measuring specific DIRE in the serum of patients enables conclusions on the particular and unique cell type that underwent necrosis. These pieces of information might add to the overall patient assessment on an intensive care unit and might help regulate vital signs or to specifically react to a sudden hormone release at supraphysiological concentrations.

immune responses to regulated cell death becomes clear given the two inevitable consequences of cellular necrosis (BOX 2).

\section{Apoptosis versus necrosis. Apoptosis} represents the default pathway of cellular turnover in higher organisms ${ }^{126}$. It does not cause pro-inflammatory reactions and the dying cells expose phosphatidylserine on the cell surface to ensure the rapid removal by macrophages ${ }^{126}$. Whereas apoptosis does not drive an active inflammatory response, and in fact the engulfment of apoptotic cells results in the production of anti-inflammatory cytokines (BOX 3), necrotic cell death results in a DAMP-driven immune response. Intracellular antigens or neo-antigens exposed on membranes of damaged organelles or simply on large enough structures, such as the cytoskeleton or protein-signalling complexes, might trigger naive cells of the adaptive immune system and even cause the generation of antibodies. In mouse models of systemic lupus erythematosus, the failure to remove necrotic debris is associated with the production of anti-nuclear antibodies and auto-antibodies against double-stranded DNA $^{127-129}$. Such consequences of necrotic cell death apply to all pathways of regulated and even traumatic necrosis.

Necroptosis. The release of IL-33 (REF. ${ }^{130}$ ), referred to by some authors as a 'necroptotic DAMP'131, results in the local activation of ST-2 receptors such as on the surface of regulatory $\mathrm{T}$ cells ${ }^{132}$. Thus, IL-33 signalling might result in the prominent appearance of such cells in the microenvironment surrounding a cell course that died by necroptosis ${ }^{132}$. However, IL-33 might also regulate the cross-priming process in dendritic cells ${ }^{130}$, but further work is needed to conclude whether such processes are clearly pro-inflammatory. The additional release of CXCL1 from necroptotically dying cells ${ }^{133}$ might inhibit infiltrating neutrophils (as opposed to neutrophil recruitment during ferroptosis). If necroptosis is interpreted as a defence mechanism against apoptosis-inhibiting viruses, it conceptually makes sense that the inflammatory response is limited to the microenvironment. However, necroptosis in gut stem cells accompanied with the loss of the gastrointestinal barrier function, induced by the absence of the transcription factor SETDB1, was sufficient to drive severe inflammatory bowel disease ${ }^{134,135}$. Along similar lines, an MLKL-missense mutation results in lethal neonatal inflammation ${ }^{136}$. 
Pyroptosis. In the case of caspase

1 -activating inflammasomes, IL- $1 \beta$ and IL-18

(two long-lasting cytokines) are released after maturation upon pyroptosis in addition to DAMPs and DIRE ${ }^{137,138}$. This does not appear to be the case if only caspase 11 is activated, which leads to GSDMD cleavage ${ }^{81}$ but not to maturation of the cytokines. Caspase 1-driven pyroptosis has been best described for activated monocytes and it remains unclear if induction of pyroptosis in, for instance, epithelial or malignant cells results in a similar pro-inflammatory signature ${ }^{81,139-141}$. Therefore, with respect to the immunogenicity of pyroptosis, the distinction between pyroptosis mediated by caspase 1 and caspase 11 or pyroptosis mediated by caspase 11 alone is obvious. In contrast to all other regulated cell death pathways, caspase 1-driven pyroptosis includes IL- $1 \beta$ and IL-18 release to fight intracellular bacteria. Clinically, these cytokines are associated with an increase in body temperature (fever) based on cytokine signalling in the hypothalamus ${ }^{142}$.

Ferroptosis. The best-studied response to synchronized regulated necrosis mediated by ferroptosis is the infiltration of neutrophils. Within 2 hours following reperfusion of a cardiac transplant, massive infiltration of the tissue by neutrophils was demonstrated by intravital microscopy ${ }^{143}$. Similarly, synchronized regulated necrosis in the kidney tubules (FIG. 2) is associated with a marked neutrophil activation ${ }^{144,145}$. However, to the best of our knowledge, the inflammatory signature of neutrophils in classic endocrine organs undergoing ferroptosis has not been tested and the recruiting factors remain to be identified. Some reports investigated short-lived lipid peroxides but this approach, like all others, does not allow the identification of a specific signal that might be therapeutically $\operatorname{targeted~}^{91,143}$. Other features of ferroptosisinduced necroinflammation (for example, dendritic cell priming and naive $B$ cell priming) are subject to investigation and it will be interesting to work out the concise interconnection between ferroptosis and the immune system.

\section{Pharmacological modulation}

During the past decade, medicinal chemists have developed several inhibitors or tried to repurpose existing drugs to prevent regulated necrosis ${ }^{146,147}$. Generally, inhibitors of necroptosis are referred to as 'necrostatins' and inhibitors of ferroptosis are known as 'ferrostatins'. However, to the best of our knowledge, the term 'pyrostatins' has not

\section{Box $3 \mid$ Steroids and regulated cell death}

Clinically, tumour lysis syndrome is a severe complication of the chemotherapy used for haematological cancers associated with the release of damage-associated molecular patterns (DNA, phosphate, potassium and cytokines). There are reports that clearly indicate that the application of dexamethasone on its own, without the often-prescribed DNA-damaging therapy, is sufficient to cause massive increases of $\mathrm{LDH}$ in patients ${ }^{224}$. From a cell death point of view, the induction of tumour lysis syndrome suggests steroid-induced necrosis (lysis is a word that can be used synonymously with necrosis). A careful review of the literature indicated that no specific necrotic pathway has been ascribed to this particular syndrome, no receptor has been identified and no alternative treatment has been suggested ${ }^{224}$. Understanding why the tumour cells are extraordinarily sensitive to necrosis in this scenario might help in developing more on-target therapeutic approaches.

been used and inhibitors of apoptosis are generally referred to as 'caspase inhibitors'. By contrast, inducers of regulated cell death, such as the apoptosis inducer venetoclax ${ }^{148}$, have been added to the clinical routine for the treatment of haematological cancers and their therapeutic profile is expected to expand. Many necroptosis, pyroptosis and FINs are being developed ${ }^{122,149}$. For the purpose of this Perspective and with respect to clinical applicability, in our opinion, the pharmacological modulation of necroptosis and ferroptosis represents the most promising novel therapeutic approach to prevent inflammatory cell loss in a variety of diseases, such as T1DM.

Necrostatins, such as the small molecule necrostatin 1 (REF. ${ }^{150}$ ), have boosted the research in regulated necrosis due to their high specificity to RIPK1 kinase inhibition $^{151}$. It was demonstrated that the original compound of necrostatin 1 , in addition to targeting RIPK1, has some inhibitory effect against ferroptosis ${ }^{105}$ that can be explained by the reducing activity of a thiohydantoin in the structure of necrostatin 1 (REF. ${ }^{147}$ ). This finding has caused confusion in the cell death field; however, with the development of more specific inhibitors of the RIPK1 kinase domain (that is, necrostatin 1s) ${ }^{151}$ and the development of RIPK1 kinase-dead knock-in mice ${ }^{152-154}$, this problem was resolved. Inhibitors of RIPK3 $\left(\right.$ REF. $^{155}$ ) and MLKL ${ }^{5}$ also exist and it might be most efficient to inhibit necroptosis by simultaneously targeting multiple steps of the pathway ${ }^{156}$.

The inhibition of ferroptosis might be of paramount therapeutic interest as it was demonstrated within the past decade to be the fundamentally important necrotic cell death mechanism that drives myocardial infarction-induced cardiomyocyte necrosis $^{143,157-159}$, (haemorrhagic) stroke $^{160,161}$, acute kidney injury ${ }^{96,162-165}$ and liver failure ${ }^{104,105}$. In addition, it seems to account for a marked amount of the necrotic content transplanted upon solid organ transplantation ${ }^{2,143,166}$. Inhibitors of ferroptosis (ferrostatins) are readily available for preclinical research and it will be exciting to see their transfer to clinical trials ${ }^{146,167}$. Specific ferroptosis inhibitors, such as ferrostatin 1 (REF. ${ }^{107}$ ), 16-86 (a novel third-generation ferrostatin $)^{96}$, liproxstatin 1 $\left(\right.$ REF. $^{105}$ ) or UCAM-3203/3206 (REF. ${ }^{168}$ ), allowed the detection of the broad clinical importance of ferroptosis in cardiovascular diseases but hardly any endocrine disorders have been investigated with these novel inhibitors. By contrast, the four classes of FINs are widely used. Class 1 FINs (such as erastin) inhibit system $\mathrm{x}_{\mathrm{c}}^{-}\left(\mathrm{REF}^{169}\right)$, whereas class 2 FINs (such as RSL3) directly target the active centre of GPX4 (REF. ${ }^{106}$ ). FIN56, the prototype class 3 FIN, functions by inducing the degradation of GPX4 $\left(\mathrm{REF}^{118}\right)$. Finally, the precise mechanism of FINO2 as a class 4 FIN has not been fully identified $^{119}$. In addition, inhibitors of the FSP1 oxidoreductase ${ }^{108,109}$ or thioredoxin, such as ferroptocide ${ }^{149}$, offer even more options to drive cells into ferroptosis. It will be interesting to see cell lines of endocrine malignancies treated with FINs of all classes as a road to novel therapies.

The translational potential of an expanded understanding of regulated necrosis might not be limited to the inhibition of cell death and necroinflammation. By contrast, cancers, including adrenocortical carcinomas in particular, upregulate anti-ferroptosis proteins such as GPX4 (REF. $\left.{ }^{10}\right)$. This dependency provides a selective advantage to the tumour cells to more potently resist lipid peroxidation that originates, for example, from mitochondria in cells that consume high energy levels ${ }^{106,123}$. However, GPX4 expression, in the case of adrenocortical carcinomas, is so much higher than in normal tissue that a ferroptosis-inducing strategy might be considered. One such compound, imidazole ketone erastin, did not exhibit a strong adverse effect profile in preclinical testing 
in rodents ${ }^{122}$. As it is expected that more endogenous anti-ferroptosis mechanisms will be discovered, such as FSP1, more therapeutic options might emerge.

The induction of necrotic cell death does not necessarily need to be limited to ferroptosis induction. Necroptosis was demonstrated to improve the $\mathrm{T}$ cell cross-priming mediated by dendritic cells that process necroptotic debris ${ }^{3}$. Indeed, necroptosis seems to be a tightly regulated pro-inflammatory signalling pathway ${ }^{170}$ not only because of the release of DAMPs ${ }^{171}$. These fundamental concepts led to the model of necroinflammation ${ }^{2,172}$, which can be exploited to boost cancer immunotherapy ${ }^{171,173,174}$ in general and in endocrine tumours in particular.

\section{Cell death in endocrine disorders}

It is beyond the scope of this Perspective to list all the endocrine disorders that might be associated with regulated cell death. The listed disorders were chosen based on our opinion on the strength of the evidence on cell death as a causative factor. Supplementary Fig. 1 provides a subjective overview of the selected endocrine organs and cell death pathways that might be involved. Supplementary Table 1 summarizes some of the previously published literature on cell death in endocrine disorders and is interpreted in light of the current knowledge on regulated necrosis.

Cell death in diabetes mellitus. Several stages of classic T1DM are pathophysiologically driven by necrosis pathways (BOX 4;
Supplementary Fig. 2). A paper published in 2015 demonstrated that autoreactive $\mathrm{T}$ cells do not induce classic apoptosis or necroptosis in pancreatic $\beta$-cells but instead cause another pathway of necrosis ${ }^{9}$. These reports are in line with the results of scientific attempts to induce FAS ligand (CD95L and CD178) expression in murine $\beta$-cells ${ }^{175}$, which resulted in a rapid destruction caused by neutrophil infiltration ${ }^{176}$. Given the evidence of ferroptosis to induce neutrophil infiltration within no more than 2 hours following cardiac transplantation ${ }^{143}$, we consider ferroptosis a possible mechanism of $\beta$-cell death. However, the mechanisms of how $\mathrm{T}$ cells drive a cell into ferroptosis are unclear (BOX 4; Supplementary Fig. 2). With respect to the complications of T1DM-driven atherosclerosis, the evidence is clearer. Given the evidence on the role of necroptosis and ferroptosis in myocardial infarction ${ }^{157}$, such redox systems also apply more specifically during cardiac remodelling and diabetic cardiomyopathy ${ }^{177}$, implying that a hydrogen sulfide-based therapeutic strategy could be used to mitigate myocardial cell death in diabetic cardiomyopathy ${ }^{177}$. Specific necroptosis-deficient mice ( $M L K L$ knockout and/or RIPK1 kinase-dead knock-in) should be used in the future to confirm the published results.

\section{Pancreatic islet transplantation and}

ferroptosis. In 2018, we demonstrated that pancreatic islets undergo massive ferroptosis upon isolation from donors in standard media, whereas the addition of ferrostatins as small molecules

\section{Box 4 | The role of necroptosis and ferroptosis during T1DM disease progression}

Type 1 diabetes mellitus (T1DM) is caused by the loss of insulin-producing $\beta$-cells. If this is mediated by cytotoxic T cells, the most likely mode of death induction is FAS-induced apoptosis of the $\beta$-cells. However, other modes of cell death, such as necroptosis, cannot be excluded given the currently available evidence. It is well established that T1DM contributes to the pathogenesis of atherosclerosis; the pathophysiology of atherosclerosis includes necrosis in atherosclerotic plaques. This necrotic cell death might be potentially driven by cholesterol crystal-induced necroptosis but ferroptosis might also contribute. Other secondary T1DM complications are driven by non-enzymatic glycosylation and result in the dysfunction rather than cell death of neurons (for example, diabetic retinopathy and diabetic neuropathy). However, in the kidney (diabetic nephropathy), acute tubular necrosis (mainly driven by ferroptosis) is common in patients with T1DM. Later in the course of T1DM, cardiovascular disease might extend to heart attacks and strokes, both of which are predominantly driven by ferroptosis, especially in haemorrhagic stroke. However, solid evidence on the role of necroptosis in heart attacks exists. Finally, patients with T1DM might require the combined transplantation of the pancreas (or pancreatic islets) and kidney. During the cold storage of solid organs, both necroptosis and ferroptosis contribute to the overall amount of necrosis that is transplanted. In line with the current understanding of necroinflammation, the transplanted necrotic debris activates naive B cells. Upon immunosuppression, the proliferation of these cells is inhibited, rendering it more likely for them to obtain a memory B cell phenotype in the sense of an anti-graft vaccination. Years later, de novo donor-specific antibodies against the graft might cause antibody-mediated rejection of the transplant, typically following an episode of viral infection.

increases the transplantable number of islet equivalents ${ }^{178,179}$. Therefore, islet transplantation would potentially no longer be limited by the number of donor islets, as the cell death of $\beta$-cells can potentially be completely prevented.

As depicted in Supplementary Fig. 2, the transplantation of necrotic debris might activate naive $B$ cells that differentiate into memory B cells upon immunosuppression. Years after transplantation, these memory $B$ cells might become re-activated by the necrotic clearance of viruses in the graft cells and cause steroid-resistant antibody-mediated rejection of the transplanted tissues ${ }^{2,180}$. Experimentally, ferroptosis-deficient ACSL4-knockout pancreatic $\beta$-cells should be used to confirm the role of ferroptosis in islet transplantation.

Adrenal insufficiency. Adrenal insufficiency can originate following a series of insults, such as haemorrhagic, ischaemic, infectious, surgical or metastatic destruction of the adrenal tissue ${ }^{181-183}$. Depending on the nature of the pathophysiology, ischaemic and haemorrhagic destruction can be caused by ferroptosis and necroptosis, whereas the loss of adrenal cells during viral defence might be a consequence of apoptosis or necroptosis. In the special case of acute bacterial infection, such as in WaterhouseFriedrichsen syndrome, the fulminating shock might cause ischaemic destruction but it cannot be excluded that bacteria inside the adrenal gland induce a macrophage response, including pyroptotic cell death ${ }^{184}$. In this setting, bleeding in the adrenal tissue might lower the threshold for ferroptosis, given the presence of free iron from damaged erythrocytes. The adrenal tissue, therefore, exemplifies how several different cell death modes can affect the same organ ${ }^{7}$. More research is required to distinguish these modes and allocate clinical situations as indications for specific anti-cell-death treatments.

The importance of cell death in adrenal tissue additionally becomes obvious given the fact that Addison disease results from the loss of $>90 \%$ of adrenal tissue before clinical presentation. Along similar lines, inflammation-related adrenal insufficiency might be triggered by necrotic cells and necroinflammation. Upon failure to remove necrotic cells through the molecule developmental endothelial locus 1 , cell death, adrenal insufficiency and inflammation in adrenal tissue were exacerbated ${ }^{185}$. However, it remains unclear how the majority of adrenal cells die during 
the progression of adrenal insufficiency. Future research might consider the use of ferrostatins, necrostatins and specific genetic models ( $M L K L$ knockout or ACSL4-conditional deficiency in adrenal tissue) to confirm the preliminary findings.

\section{Cell death in hypoparathyroidism.}

Autosomal dominant familial isolated hypoparathyroidism is an orphan disease that is caused by TUNEL-positive cell death ${ }^{186}$. Mechanistically, mutations result in a non-functional human preproparathyroid hormone that is trapped in the ER before the release process can start ${ }^{186}$. It remains unclear if the resulting ER stress triggers necrotic cell death, for example, by ferroptosis or necroptosis. Importantly, expression upon co-incubation with 4-phenylbutyric acid prevents the misfolding of preproparathyroid hormone and the death of the cell. Additionally, the secretion of the mutated hormone was shown to be restored. Other reports concluded that 4-phenylbutyric acid supplementation prevents ER stress and reduces levels of 4-hydroxy-trans-2-nonenal and lipid peroxidation ${ }^{187}$. However, ER stress has also been implicated as an originating factor of ferroptotic cell death ${ }^{188}$.

Disorders of the thyroid gland. Radioiodine therapy drives necrotic cell death in thyroid adenomas and Graves disease as well as in malignant tumours of the thyroid ${ }^{189}$. In clinical practice, 131-iodine therapy is associated with DIRE (in this case initial hyperthyroidism followed by thyroid failure) and can therefore be prevented by the administration of peroxidase inhibitors of the thioamide family, such as carbimazole. Interestingly, a known adverse effect of radioiodine therapy is irradiationassociated thyroiditis. Along similar lines, one report suggested that excessive iodine therapy promotes pyroptosis in Hashimoto thyroiditis ${ }^{190}$. In conclusion, radiation and radioiodine treatment of thyroid follicular epithelial cells can cause follicular cell necrosis and thereby drive necroinflammation and DIRE.

Primary thyrotoxicosis (associated with high free tetra-iodothyronine $\left(\mathrm{T}_{4}\right)$ and low TSH) is a prototype complication caused by the release of DIRE, in this case $\mathrm{T}_{4}$. By contrast, tri-iodothyronine $\left(\mathrm{T}_{3}\right)$ toxicosis clinically appears as a special condition but pathophysiologically also includes $\mathrm{T}_{3}$-DIRE release. Through similar mechanisms, thyrotoxicosis might occur secondary to an infarcted thyroid adenoma ${ }^{191}$ and simply any condition that causes thyroid cell necrosis.
Another thyroid disease with a high release of thyroid hormone DIRE is the painful subacute thyroiditis called De Quervain syndrome. This inflammatory thyroid disease with a potential viral aetiology background presents with clinical laboratory manifestations of local and systemic inflammation, including elevated levels of $\mathrm{TNF}^{192}$. In the early phase, there is a destruction of follicular epithelial cells with colloid extravasation and infiltration of follicles predominately by neutrophils forming micro-abscesses. A tri-phasic endocrine manifestation is characterized by initial thyroid hormone release inducing thyrotoxicosis, followed by thyroid destruction-induced hypothyroidism and eventually a return to normal conditions with euthyroidism. Cell death has been implicated in the pathophysiology of hypothyroidism ${ }^{192}$ but a detailed analysis of the specific mechanism is lacking.

Growth hormone deficiency. Isolated autosomal dominant growth hormone deficiency (IGHD) has been associated with increased cellular proliferation and apoptosis ${ }^{193}$. ER stress might be a driver of IGHD $^{194}$, associated with complex patterns of caspase activation ${ }^{194}$ that might indicate crosstalk to the necroptosis pathway. Finally, it was demonstrated that $\mathrm{GH}$ inhibits $\mathrm{p} 53$ in colon carcinomas ${ }^{195}$, indicating that $\mathrm{GH}$ might regulate the ferroptosis sensitivity that might also play a role in pituitary cells. Clearly, more work on cell death, for example, through the use of ferrostatins and necrostatins, is needed to clarify which pathway most prominently causes IGHD.

Primary aldosteronism. Aldosterone was reported to induce cell death in aortic cells, a result that was interpreted as apoptosis at this time ${ }^{196}$. Somatic mutations in aldosterone-producing adenomas can induce cell death. In particular, higher percentages of dead cells were observed upon KCNJ5-G151R, G151E and L168R mutations under conditions tested with the incubation of cumate ${ }^{197}$ when compared with cells carrying the KCNJ5T158A mutation. Interestingly, these observations were associated with the immunofluorescence detection of cleaved PARP, a downstream molecule that carries a caspase cleavage site and was therefore often interpreted as apoptosis. This nuclear staining for positive cleaved PARP in the nuclei of KCNJ5-G151E-transfected and L168R-transfected cells was significantly higher than in mock-transfected controls. These data indicate that apoptosis might be caused by aldosterone mutants, although it is unclear how the apoptotic pathway might be activated in such conditions.

Hypogonadism. Leydig cells are particularly sensitive to the induction of cell death by oxygen radicals and an important role for peroxiredoxin 2 has been discovered to prevent this form of cell death ${ }^{198}$. The sensitive cells in this study exhibited a markedly lower testosterone production, but experimentally it remains unclear if this effect is due to less cellular mass following cell loss ${ }^{198}$. In primordial germ cells, DNA repair is of particular importance. Fanconi anaemia is associated with hypogonadism and is caused by germline mutations in DNA repair pathways, such as p53-p21. Interestingly, a study published in 2014 indicated that hypogonadism in patients with Fanconi anaemia is not associated with apoptosis and it was indirectly concluded that a lesser rate of proliferation might cause this syndrome ${ }^{199}$. However, besides its well-defined functions in proliferation and cell cycle progression ${ }^{200}$, p53 is known to be involved in the ferroptosis pathway ${ }^{120,201}$. In addition, p53 is known to regulate the mevalonate pathway, an alternative cellular source of $\mathrm{GSH}^{202}$.

\section{Haemochromatosis, iron overload states} and endocrine dysfunction. Iron-catalysed necrosis (ferroptosis) was demonstrated to be a critical mediator of the most common cardiovascular events, including heart attacks $^{143,157,158}$ and strokes ${ }^{150,160}$. In genetically predisposed patients, such as those with the C282Y mutation of the HFE gene linked to the HLA-A locus on chromosome $6 \mathrm{p}$, the liver is the most affected organ ${ }^{203}$. Direct damage to pancreatic islets, most likely induced by ferroptosis, occurs frequently in up to $65 \%$ of symptomatic patients with haemochromatosis. Other complications include cardiac arrhythmias and congestive heart failure in approximately $15 \%$ of affected patients. Hypogonadism, caused by undefined cell death, can antedate other clinical features. Interestingly, the HFE gene has been associated with far more common hepatic conditions, such as nonalcoholic steatosis ${ }^{204}$, that also might involve ferroptosis ${ }^{205,206}$.

\section{Cell death in endocrine malignancies}

As is the case with the endocrine disorders described in the previous section, it is beyond the scope of this Perspective to list all the endocrine malignancies that might be associated with regulated cell death. Please refer to Supplementary Fig. 1 for a subjective 
overview of the selected endocrine organs and cell death pathways that might be involved.

\section{Cell death in adrenocortical carcinoma.} It has been recognized for decades that adrenocortical carcinomas exhibit high levels of TUNEL positivity ${ }^{207}$ (BOX 1) and that mutations in the tumour suppressor p53 frequently occur ${ }^{208}$, although not in all populations in the same manner ${ }^{209}$. However, in 2019, we discovered the exquisite sensitivity of adrenocortical carcinomas to the induction of ferroptosis ${ }^{10}$, a necrotic-type cell death that explains both TUNEL positivity and regulation by $\mathrm{p} 53$. Interestingly, in contrast to GPX4, members of the glutathione peroxidase family were found amongst the most frequently mutated genes in adrenocortical carcinomas (encoding GPX3, GPX7 and GPX8) 210,211. The same studies also found lipoxygenases, such as ALOX12, to be mutated, potentially as a tumour strategy to evade ferroptotic cell death. Along similar lines, thioredoxin reductases were overexpressed, potentially as a means to avoid lipid peroxidation ${ }^{210,211}$. Indeed, thioredoxin inhibition by a compound named ferroptocide was reported to drive cancer cells into ferroptosis ${ }^{149}$; however, this has not been investigated in adrenocortical carcinomas so far. In addition, other strategies that specifically target endocrine cancers using imidazole ketone erastin (a FIN) exist ${ }^{122}$ and will be developed further ${ }^{121}$. Currently, these efforts are at an experimental preclinical stage.

\section{Cell death in phaeochromocytoma and} paragangliomas. DNA methylation profiling in phaeochromocytoma and paraganglioma revealed GPX3 as a frequently mutated prognostic marker ${ }^{212}$. It is nevertheless unclear if and how GPX3 functions to regulate ferroptosis. However, the evidence for ferroptosis is weak compared with that for adrenocortical carcinomas. One early study demonstrated 7 of 29 (24\%) phaeochromocytomas to be associated with the loss of heterozygosity on $17 \mathrm{p}$, a DAMP release

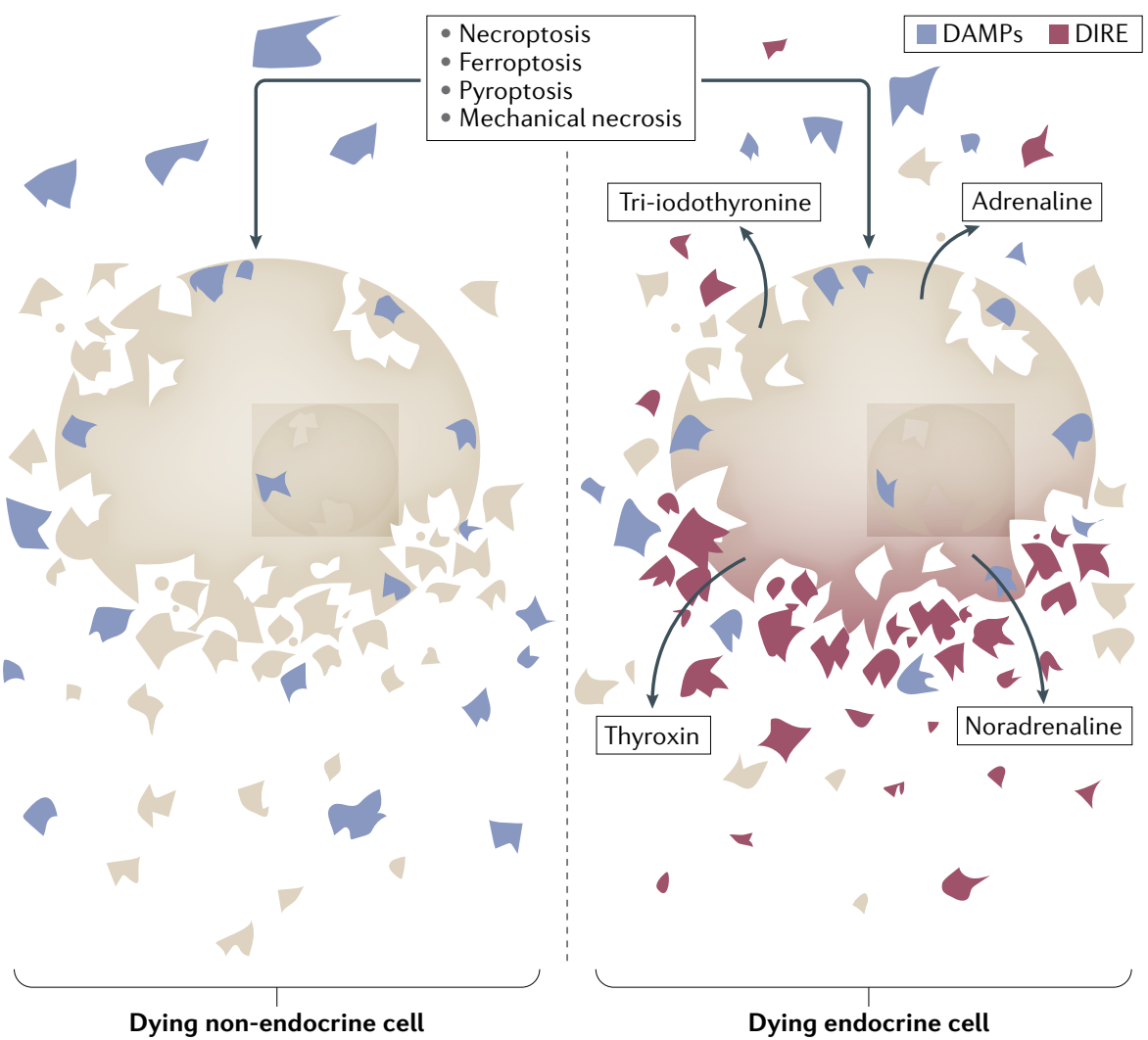

Fig. 3 | DAMPs and DIRE and the consequences of cell death. Regulated necrosis (necroptosis, ferroptosis and pyroptosis) and non-regulated necrosis, such as mechanical manipulation of tissue, results in the release of damage-associated molecular patterns (DAMPs) in non-endocrine cells (part a). By contrast, endocrine cells that die by necrosis are associated with the release of DAMPs and damage-induced release of endocrine factors (DIRE) (part b). Such factors include common hormones, such as cortisol, adrenaline, noradrenaline, thyroxin, tri-iodothyronine and aldosterone.

the locus associated with p53 $\left(\right.$ REF. $\left.^{213}\right)$. More research is necessary regarding models of phaeochromocytoma, for example, 3D human tumour models that contain a necrotic core ${ }^{214}$, to understand the nature of this cell death. It is clear that phaeochromocytomas are hypervascularized tumours with a central necrotic zone that can even be detected radiologically, referred to as the 'ring sign' ${ }^{215}$. With respect to the release of catecholamines from necrotic phaeochromocytomas, another example of DIRE release (FIG. 3), it was noted that myocardial necrosis can be associated with adrenaline release ${ }^{216}$. In extreme cases, pheochromocytoma DIRE might lead to severe and eventually lethal shock $^{217,218}$. Likewise, it is worth mentioning that, as early as 1961, a case report pointed to a potentially mechanistic link between phaeochromocytoma and acute tubular necrosis ${ }^{219}$, the potential mechanism of which remained entirely unclear.

\section{Ferroptosis and thyroid cancer. The} targeting the pentose phosphate pathway in thyroid cancer cells was demonstrated to be associated with increased cell death dependent on the reactive oxygen species load, a typical feature of ferroptosis ${ }^{220}$.

An association with ER stress has also been linked to ferroptosis ${ }^{188}$. Along similar lines, neutrophil gelatinase-associated lipocalin (NGAL), an iron scavenging NF- $\kappa \mathrm{B}$ target protein, was identified to function as a survival factor for thyroid cancers ${ }^{221}$. These data led to the hypothesis that thyroid cancers evade ferroptosis to promote cancer survival. However, given the paucity of easyto-assess biomarkers of ferroptosis, specific ferroptosis-inhibitors and GPX4-deficient genetic models will enable the confirmation of the role of ferroptosis in thyroid cancer.

\section{Conclusions}

We are only just beginning to understand the role of regulated necrosis in endocrine disorders. As endocrinologists, we are still struggling to understand many of the fundamental unique features of the endocrine systems and a number of open questions and obstacles remain.

First, the endocrine system is characterized by an extraordinary ability to respond to internal and external stimuli with impressive adaptive cellular and structural changes. Second, benign endocrine lesions and nodules are more frequent than in any other tissue, while malignant tumour formation in endocrine glands is a rare event. Third, the vulnerability of some endocrine tissues to rapid cellular 
degeneration, such as the adrenal cortex during sepsis and inflammation, is hardly understood but clearly associated with cellular turnover. Fourth, the susceptibility of some endocrine tissues to autoimmune diseases, such as T1DM or Hashimoto disease, cannot be explained in detail, especially not with respect to the other glands that are rarely affected by immune destruction. Fifth, the role of regulated cell death has hardly been investigated in some of the more common endocrine disorders, such as all forms of diabetes mellitus, obesity, hyperlipidaemia, Graves disease and primary hyperaldosteronism. The investigation of regulated necrosis in such diseases will probably further expand the future options for clinical applications. Finally, we cannot currently sufficiently explain the relatively easy (auto) transplantability of some endocrine organs, such as parathyroids or islets, whereas other endocrine tissues, such as testes or adrenal tissue, completely lose their functions in a foreign organ environment. We believe that a molecular understanding of regulated cell death and necroinflammation will help to answer these open questions and enable the development of novel therapeutic strategies from this research area.

Wulf Tonnus (iD) 1,9 , Alexia Belavgeni (D) ${ }^{1,9}$

Felix Beuschlein (iD ${ }^{2,3}$, Graeme Eisenhofer ${ }^{4}$

Martin Fassnacht (iD) ${ }^{5}$, Matthias Kroiss (iD ${ }^{5}$

Nils P. Krone ${ }^{4,6}$, Martin Reincke (D) ${ }^{2}$,

Stefan R. Bornstein ${ }^{3,4,7}$ and Andreas Linkermann (iD) ${ }^{1,8} \otimes$

${ }^{\prime}$ Clinic of Internal Medicine III, Division of Nephrology, University Hospital Carl Gustav Carus at the Technische Universität Dresden, Dresden, Germany.

${ }^{2}$ Medizinische Klinik und Poliklinik IV, Hospital of the Ludwig-Maximilian-University Munich, Munich, Germany.

${ }^{3}$ Klinik für Endokrinologie, Diabetologie und Klinische Ernährung, Universitätsspital Zürich, Zürich, Switzerland.

${ }^{4}$ Clinic of Internal Medicine III, University Hospital Carl Gustav Carus at the Technische Universität Dresden, Dresden, Germany.

${ }^{5}$ Clinic of Internal Medicine I, Division of Endocrinology and Diabetology, University Hospital, University of Würzburg, Würzburg, Germany.

${ }^{6}$ Academic Unit of Child Health, Department of Oncology and Metabolism, University of Sheffield, Sheffield, UK.

'Diabetes and Nutritional Sciences, King's College London, London, UK.

${ }^{8}$ Biotechnology Center, Technische Universität Dresden, Dresden, Germany.

${ }^{9}$ These authors contributed equally: Wulf Tonnus, Alexia Belavgeni.

凶e-mail: andreas.linkermann@ukdd.de https://doi.org/10.1038/s41574-021-00499-w Published online 16 June 2021

1. Galluzzi, L. et al. Molecular mechanisms of cell death: recommendations of the nomenclature committee on cell death 2018. Cell Death Differ. 25, 486-541 (2018).
2. Sarhan, M., Land, W. G., Tonnus, W., Hugo, C. P. $\&$ Linkermann, A Origin and Consequences of Necroinflammation. Physiol. Rev. 98, 727-780 (2018).

3. Yatim, N. et al. RIPK1 and NF-kappaB signaling in dying cells determines cross-priming of CD8+ T cells. Science 350, 328-334 (2015).

4. Linkermann, A. et al. Two independent pathways of regulated necrosis mediate ischemia-reperfusion injury. Proc. Natl Acad. Sci. USA 110, 12024-12029 (2013).

5. Sun, L. et al. Mixed lineage kinase domain-like protein mediates necrosis signaling downstream of RIP3 kinase. Cell 148, 213-227 (2012).

6. Vanden Berghe, T. et al. Simultaneous targeting of IL- 1 and IL-18 is required for protection against inflammatory and septic shock. Am. J. Respir. Crit. Care Med. 189, 282-291 (2014).

7. Tonnus, W. et al. The clinical relevance of necroinflammation-highlighting the importance of acute kidney injury and the adrenal glands. Cell Death Differ. 26, 68-82 (2019).

8. Tonnus, W. \& Linkermann, A. "Death is my Heir" ferroptosis connects cancer pharmacogenomics and ischemia-reperfusion injury. Cell Chem. Biol. 23 202-203 (2016).

9. Zhao, Y. et al. Autoreactive T cells induce necrosis and not BCL-2-regulated or death receptor-mediated apoptosis or RIPK3-dependent necroptosis of transplanted islets in a mouse model of type 1 diabetes. Diabetologia 58, 140-148 (2015).

10. Belavgeni, A. et al. Exquisite sensitivity of adrenocortical carcinomas to induction of ferroptosis. Proc. Natl Acad. Sci. USA 116, 22269-22274 (2019).

11. Nagata, S. Apoptosis and autoimmune diseases. Ann. NY Acad. Sci. 1209, 10-16 (2010)

12. Segawa, K. \& Nagata, S. An apoptotic 'eat me' signal: phosphatidylserine exposure. Trends Cell Biol. 25 639-650 (2015)

13. Hanayama, R. et al. Identification of a factor that links apoptotic cells to phagocytes. Nature 417, 182-187 (2002).

14. Suzuki, J., Denning, D. P., Imanishi, E., Horvitz, H. R. $\&$ Nagata, S. Xk-related protein 8 and CED-8 promote phosphatidylserine exposure in apoptotic cells. Science 341, 403-406 (2013).

15. Zargarian, S. et al. Phosphatidylserine externalization, "necroptotic bodies" release, and phagocytosis during necroptosis. PLoS Biol. 15, e2002711 (2017).

16. Krammer, P. H., Arnold, R. \& Lavrik, I. N. Life and death in peripheral T cells. Nat. Rev. Immunol. 7 532-542 (2007)

17. Ofengeim, D. \& Yuan, J. Regulation of RIP1 kinase signalling at the crossroads of inflammation and cell death. Nat. Rev. Mol. Cell Biol. 14, 727-736 (2013).

18. Vanden Berghe, T., Linkermann, A., Jouan-Lanhouet, S. Walczak, H. \& Vandenabeele, P. Regulated necrosis: the expanding network of non-apoptotic cell death pathways. Nat. Rev. Mol. Cell Biol. 15, 135-147 (2014).

19. Lafont, E et al. TBK1 and IKKepsilon prevent TNF-induced cell death by RIPK1 phosphorylation. Nat. Cell Biol. 20, 1389-1399 (2018).

20. Newton, K. et al. Cleavage of RIPK 1 by caspase- 8 is crucial for limiting apoptosis and necroptosis. Nature 574, 428-431 (2019).

21. Srinivasula, S. M. Ahmad, M. Fernandes-Alnemri, T, Litwack, G. \& Alnemri, E. S. Molecular ordering of the Fas-apoptotic pathway: the Fas/APO-1 protease Mch5 is a CrmA-inhibitable protease that activates multiple Ced-3/ICE-like cysteine proteases. Proc. Natl Acad. Sci. USA 93, 14486-14491 (1996).

22. Newton, K. et al. Activity of caspase-8 determines plasticity between cell death pathways. Nature $\mathbf{5 7 5}$ 679-682 (2019).

23. Dondelinger, Y., Darding, M., Bertrand, M. J. $\delta$ Walczak, H. Poly-ubiquitination in TNFR1-mediated necroptosis. Cell Mol. Life Sci. 73, 2165-2176 (2016).

24. Peltzer, N., Darding, M. \& Walczak, H. Holding RIPK1 on the ubiquitin leash in TNFR1 signaling. Trends Cell Biol. 26, 445-461 (2016)

25. Walczak, H. TNF and ubiquitin at the crossroads of gene activation, cell death, inflammation, and cancer. Immunol. Rev. 244, 9-28 (2011)

26. Czabotar, P. E., Lessene, G., Strasser, A. \& Adams, J. M Control of apoptosis by the BCL-2 protein family: implications for physiology and therapy. Nat. Rev. Mol. Cell Biol. 15, 49-63 (2014)

27. Acehan, D. et al. Three-dimensional structure of the apoptosome: implications for assembly, procaspase- 9 binding, and activation. Mol. Cell 9, 423-432 (2002).
28. Pop, C., Timmer, J., Sperandio, S. \& Salvesen, G. S The apoptosome activates caspase- 9 by dimerization Mol. Cell 22, 269-275 (2006)

29. Zou, H., Li, Y., Liu, X. \& Wang, X. An APAF-1. cytochrome c multimeric complex is a functional apoptosome that activates procaspase-9. J. Biol. Chem. 274, 11549-11556 (1999).

30. Kaiser, W. J., Upton, J. W. \& Mocarski, E. S. Viral modulation of programmed necrosis. Curr. Opin. Virol. 3, 296-306 (2013).

31. Mocarski, E. S., Upton, J. W. \& Kaiser, W. J. Viral infection and the evolution of caspase 8-regulated apoptotic and necrotic death pathways. Nat. Rev. Immunol. 12, 79-88 (2011)

32. Lalaoui, N. et al. Mutations that prevent caspase cleavage of RIPK1 cause autoinflammatory disease. Nature 577, 103-108 (2020).

33. Tao, P. et al. A dominant autoinflammatory disease caused by non-cleavable variants of RIPK 1 . Nature 577, 109-114 (2020).

34. Kaiser, W. J. et al. RIP3 mediates the embryonic lethality of caspase-8-deficient mice. Nature $\mathbf{4 7 1}$ 368-372 (2011)

35. Oberst, A. et al. Catalytic activity of the caspase8-FLIP(L) complex inhibits RIPK3-dependent necrosis. Nature 471, 363-367 (2011).

36. Li, J. et al. The RIP1/RIP3 necrosome forms a functional amyloid signaling complex required for programmed necrosis. Cell 150, 339-350 (2012).

37. Mompean, M. et al. The structure of the necrosome RIPK1-RIPK3 Core, a human hetero-amyloid signaling complex. Cell 173, 1244-1253.e10 (2018).

38. Kaiser, W. J., Upton, J. W. \& Mocarski, E. S. Receptorinteracting protein homotypic interaction motifdependent control of NF-kappa B activation via the DNA-dependent activator of IFN regulatory factors. J. Immunol. 181, 6427-6434 (2008).

39. Rebsamen, M. et al. DAI/ZBP1 recruits RIP1 and RIP3 through RIP homotypic interaction motifs to activate NF-kappaB. EMBO Rep. 10, 916-922 (2009).

40. Upton, J. W., Kaiser, W. J. \& Mocarski, E. S. DAI/ZBP1/ DLM-1 complexes with RIP3 to mediate virus-induced programmed necrosis that is targeted by murine cytomegalovirus vIRA. Cell Host Microbe 11, 290-297 (2012).

41. Kuriakose, T. et al. ZBP1/DAl is an innate sensor of influenza virus triggering the NLRP3 inflammasome and programmed cell death pathways. Sci. Immunol. 1, aag2045 (2016).

42. Lin, J. et al. RIPK 1 counteracts ZBP1-mediated necroptosis to inhibit inflammation. Nature 540 124-128 (2016).

43. Newton, K et al. RIPK1 inhibits ZBP1-driven necroptosis during development. Nature $\mathbf{5 4 0}$, 129-133 (2016).

44. Kaiser, W. J. \& Offermann, M. K. Apoptosis induced by the toll-like receptor adaptor TRIF is dependent on its receptor interacting protein homotypic interaction motif. J. Immunol. 174, 4942-4952 (2005).

45. Kaiser, W. J. et al. Toll-like Receptor 3-mediated necrosis via TRIF, RIP3 and MLKL. J. Biol. Chem. 288 31268-31279 (2013)

46. Dillon, C. P. et al. RIPK1 blocks early postnatal lethality mediated by caspase-8 and RIPK3. Cell 157 1189-1202 (2014).

47. Zhao, J. et al. Mixed lineage kinase domain-like is a key receptor interacting protein 3 downstream component of TNF-induced necrosis. Proc. Natl Acad. Sci. USA 109, 5322-5327 (2012).

48. Cho, Y. S. et al. Phosphorylation-driven assembly of the RIP1-RIP3 complex regulates programmed necrosis and virus-induced inflammation. Cell 137 1112-1123 (2009).

49. He, S. et al. Receptor interacting protein kinase-3 determines cellular necrotic response to TNF-alpha. Cell 137, 1100-1111 (2009).

50. Zhang, D. W. et al. RIP3, an energy metabolism regulator that switches TNF-induced cell death from apoptosis to necrosis. Science 325, 332-336 (2009)

51. Gong, Y.-N. et al. ESCRT-III Acts downstream of MLKL to regulate necroptotic cell death and its consequences. Cell 169, 286-300.e16 (2017).

52. Yoon, S., Kovalenko, A., Bogdanov, K. \& Wallach, D. MLKL, the protein that mediates necroptosis, also regulates endosomal trafficking and extracellular vesicle generation. Immunity 47, 51-65.e7 (2017).

53. Nogusa, S. et al. RIPK3 activates parallel pathways of MLKL-driven necroptosis and FADD-mediated apoptosis to protect against influenza a virus. Cell Host Microbe 20,13-24 (2016).

54. Karki, R. et al. Synergism of TNF- $\alpha$ and IFN- $\gamma$ triggers inflammatory cell death, tissue damage, and mortality 
in SARS-CoV-2 infection and cytokine shock syndromes. Cell 184, 149-168 e17 (2021).

55. Nakamura, H. et al. Serum levels of receptorinteracting protein kinase-3 in patients with COVID-19. Crit. Care 24, 484 (2020)

56. Hollstein, T. et al. Autoantibody-negative insulindependent diabetes mellitus after SARS-CoV-2 infection: a case report. Nat. Metab. 2, 1021-1024 (2020).

57. Vanlangenakker, N. et al. clAP1 and TAK1 protect cells from TNF-induced necrosis by preventing RIP1 RIP3-dependent reactive oxygen species production. Cell Death Differ. 18, 656-665 (2011).

58. Feoktistova, M. et al. clAPs block ripoptosome formation, a RIP1/Caspase-8 containing intracellular cell death complex differentially regulated by cFLIP isoforms. Mol. Cell 43, 449-463 (2011).

59. Peltzer, N. et al. LUBAC is essential for embryogenesis by preventing cell death and enabling haematopoiesis. Nature 557, 112-117 (2018).

60. Ikeda, F. et al. SHARPIN forms a linear ubiquitin ligase complex regulating NF-kappaB activity and apoptosis. Nature 471, 637-641 (2011).

61. Haas, T. L. et al. Recruitment of the linear ubiquitin chain assembly complex stabilizes the TNF-R 1 signaling complex and is required for TNF-mediated gene induction. Mol. Cell 36, 831-844 (2009).

62. Onizawa, M. et al. The ubiquitin-modifying enzyme A20 restricts ubiquitination of the kinase RIPK3 and protects cells from necroptosis. Nat. Immunol. 16, 618-627 (2015)

63. Wertz, I. E. et al. De-ubiquitination and ubiquitin ligase domains of A20 downregulate NF-kappaB signalling. Nature 430, 694-699 (2004).

64. Hrdinka, M. et al. CYLD limits Lys63- and Met1-linked ubiquitin at receptor complexes to regulate innate immune signaling. Cell Rep. 14, 2846-2858 (2016).

65. Moquin, D. M., McQuade, T. \& Chan, F. K. CYLD deubiquitinates RIP1 in the TNFalpha-induced necrosome to facilitate kinase activation and programmed necrosis. PloS ONE 8, e76841 (2013).

66. Trompouki, E. et al. CYLD is a deubiquitinating enzyme that negatively regulates NF-kappaB activation by TNFR family members. Nature 424, 793-796 (2003).

67. Kovalenko, A. et al. The tumour suppressor CYLD negatively regulates NF-kappaB signalling by deubiquitination. Nature 424, 801-805 (2003).

68. Heger, K. et al. OTULIN limits cell death and inflammation by deubiquitinating LUBAC. Nature 559, 120-124 (2018).

69. Damgaard, R. B. et al. The deubiquitinase OTULIN is an essential negative regulator of inflammation and autoimmunity. Cell 166, 1215-1230 (2016).

70. Keusekotten, K. et al. OTULIN antagonizes LUBAC signaling by specifically hydrolyzing Met 1 -linked polyubiquitin. Cell 153, 1312-1326 (2013).

71. Xu, D. et al. TBK1 suppresses RIPK 1-driven apoptosis and inflammation during development and in aging. Cell 174, 1477-1491.e19 (2018).

72. Dondelinger, Y. et al. RIPK3 contributes to TNFR1 mediated RIPK1 kinase-dependent apoptosis in conditions of CIAP $1 / 2$ depletion or TAK 1 kinase inhibition. Cell Death Differ. 20, 1381-1392 (2013).

73. Dondelinger, Y. et al. NF-kappaB-independent role of IKKalpha/IKKbeta in preventing RIPK1 kinasedependent apoptotic and necroptotic cell death TNF signaling. Mol. Cell 60, 63-76 (2015)

74. Annibaldi, A. et al. Ubiquitin-mediated regulation of RIPK1 kinase activity Independent of IKK and MK2 Mol. Cell 69, 566-580.e5 (2018)

75. Jaco, I. et al. MK2 phosphorylates RIPK1 to prevent TNF-induced cell death. Mol. Cell 66, 698-710.e5 (2017).

76. Mihaly, S. R., Ninomiya-Tsuji, J. \& Morioka, S TAK 1 control of cell death. Cell Death Differ. 21 1667-1676 (2014)

77. de Vasconcelos, N. M. Van, O. N. \& Lamkanfi, M. Inflammasomes as polyvalent cell death platforms Cell Mol. Life Sci. 73, 2335-2347 (2016)

78. Broz, P. \& Dixit, V. M. Inflammasomes: mechanism of assembly, regulation and signalling. Nat. Rev. Immunol. 16, 407-420 (2016).

79. Ding, J. et al. Pore-forming activity and structural autoinhibition of the gasdermin family. Nature $\mathbf{5 3 5}$ 111-116 (2016).

80. Kayagaki, N. et al. Caspase-11 cleaves gasdermin D for non-canonical inflammasome signaling. Nature 526, 666-671 (2015).

81. Shi, J. et al. Cleavage of GSDMD by inflammatory caspases determines pyroptotic cell death. Nature 526, 660-665 (2015).
82. Ruan, J., Xia, S., Liu, X., Lieberman, J. \& Wu, H. Cryo-EM structure of the gasdermin A3 membrane pore. Nature 557, 62-67 (2018).

83. Fritsch, M. et al. Caspase-8 is the molecular switch for apoptosis, necroptosis and pyroptosis. Nature $\mathbf{5 7 5}$ 683-687 (2019).

84. Kang, T. B., Yang, S. H., Toth, B., Kovalenko, A. \& Wallach, D. Caspase-8 blocks kinase RIPK3-mediated activation of the NLRP3 inflammasome. Immunity 38 27-40 (2013)

85. Lawlor, K. E. et al. RIPK3 promotes cell death and NLRP3 inflammasome activation in the absence of MLKL. Nat. Commun. 6, 6282 (2015).

86. Philip, N. H. et al. Caspase-8 mediates caspase- 1 processing and innate immune defense in response to bacterial blockade of NF-kappaB and MAPK signaling Proc. Natl Acad. Sci. USA 111, 7385-7390 (2014).

87. Vince, J. E. et al. Inhibitor of apoptosis proteins limit RIP3 kinase-dependent interleukin-1 activation Immunity 36, 215-227 (2012)

88. Rogers, $\mathrm{C}$ et al. Gasdermin pores permeabilize mitochondria to augment caspase- 3 activation during apoptosis and inflammasome activation. Nat. Commun. 10, 1689 (2019).

89. Wang, Y. et al. Chemotherapy drugs induce pyroptosis through caspase-3 cleavage of a Gasdermin. Nature 547, 99-103 (2017)

90. Taabazuing, C. Y. Okondo, M. C. \& Bachovchin, D. A. Pyroptosis and apoptosis pathways engage in bidirectional crosstalk in monocytes and macrophages. Cell Chem. Biol. 24, 507-514.e4 (2017).

91. Tonnus, W. et al. The pathological features of regulated necrosis. J. Pathol. 247, 697-707 (2019).

92. Ruhl, S. et al. ESCRT-dependent membrane repair negatively regulates pyroptosis downstream of GSDMD activation. Science 362, 956-960 (2018).

93. Mandal, P et al Caspase-8 collaborates with caspase- 11 to drive tissue damage and execution of endotoxic shock. Immunity 49, 42-55.e6 (2018).

94. Orning, P. et al. Pathogen blockade of TAK 1 triggers caspase-8-dependent cleavage of gasdermin $D$ and cell death. Science 362, 1064-1069 (2018).

95. Sarhan, J. et al. Caspase-8 induces cleavage of gasdermin $D$ to elicit pyroptosis during Yersinia infection. Proc. Natl Acad. Sci. USA 115 E10888-E10897 (2018).

96. Linkermann, A. et al. Synchronized renal tubular cell death involves ferroptosis. Proc. Natl Acad. Sci. USA 111, 16836-16841 (2014).

97. Kim, S. E. et al. Ultrasmall nanoparticles induce ferroptosis in nutrient-deprived cancer cells and suppress tumour growth. Nat. Nanotechnol. 11, 977-985 (2016).

98. Riegman, M. et al. Ferroptosis occurs through an osmotic mechanism and propagates independently of cell rupture. Nat. Cell Biol. 22, 1042-1048 (2020).

99. Katikaneni, A et al. Lipid peroxidation regulates long-range wound detection through 5-lipoxygenase in zebrafish. Nat. Cell Biol. 22, 1049-1055 (2020).

100. Stockwell, B. R. et al. Ferroptosis: a regulated cell death nexus linking metabolism, redox biology, and disease. Cell 171, 273-285 (2017).

101. Conrad, M. \& Pratt, D. A. The chemical basis of ferroptosis. Nat. Chem. Biol. 15, 1137-1147 (2019).

102. Davidson, A. J. \& Wood, W. Igniting the spread of ferroptotic cell death. Nat. Cell Biol. 22, 1027-1029 (2020).

103. Seiler, A. et al. Glutathione peroxidase 4 senses and translates oxidative stress into 12/15-lipoxygenase dependent- and AIF-mediated cell death. Cell Metab. 8, 237-248 (2008).

104. Yoo, S. E. et al. Gpx4 ablation in adult mice results in a lethal phenotype accompanied by neuronal loss in brain. Free Radic. Biol. Med. 52, 1820-1827 (2012)

105. Friedmann Angeli, J. P. et al. Inactivation of the ferroptosis regulator Gpx4 triggers acute renal failure in mice. Nat. Cell Biol. 16, 1180-1191 (2014).

106. Yang, W. S. et al. Regulation of ferroptotic cancer cell death by GPX4. Cell 156, 317-331 (2014).

107. Dixon, S. J. et al. Ferroptosis: an iron-dependent form of nonapoptotic cell death. Cell 149, 1060-1072 (2012).

108. Bersuker, K. et al. The CoQ oxidoreductase FSP1 acts parallel to GPX4 to inhibit ferroptosis. Nature 575, 688-692 (2019).

109. Doll, S. et al. FSP1 is a glutathione-independent ferroptosis suppressor. Nature 575, 693-698 (2019).

110. Brown, C. W. et al. Prominin2 drives ferroptosis resistance by stimulating iron export. Dev. Cell $\mathbf{5 1}$, 575-586 (2019).
111. Dixon, S. J. et al. Human haploid cell genetics reveals roles for lipid metabolism genes in nonapoptotic cell death. ACS Chem. Biol. 10, 1604-1609 (2015).

112. Doll, S. et al. ACSL4 dictates ferroptosis sensitivity by shaping cellular lipid composition. Nat. Chem. Biol. 13, 91-98 (2017).

113. Kagan, V. E. et al. Oxidized arachidonic and adrenic PEs navigate cells to ferroptosis. Nat. Chem. Biol. 13 , 81-90 (2017)

114. Wang, W. et al. Tissue-specific ablation of ACSL4 results in disturbed steroidogenesis. Endocrinology 160, 2517-2528 (2019).

115. Arlt, W. et al. Congenital adrenal hyperplasia caused by mutant P450 oxidoreductase and human androgen synthesis: analytical study. Lancet 363, 2128-2135 (2004).

116. Soula, M. et al. Metabolic determinants of cancer cell sensitivity to canonical ferroptosis inducers. Nat. Chem. Biol. 16, 1351-1360 (2020)

117. Yan, B. et al. Membrane damage during ferroptosis is caused by oxidation of phospholipids catalyzed by the oxidoreductases POR and CYB5R1. Mol. Cell 81 355-369.e10 (2021)

118. Shimada, K. et al. Global survey of cell death mechanisms reveals metabolic regulation of ferroptosis. Nat. Chem. Biol. 12, 497-503 (2016).

119. Gaschler, M. M. et al. FINO2 initiates ferroptosis through GPX4 inactivation and iron oxidation. Nat. Chem. Biol. 14, 507-515 (2018).

120. Jiang, L. et al. Ferroptosis as a p53-mediated activity during tumour suppression. Nature 520, 57-62 (2015).

121. Yi, J., Minikes, A. M. \& Jiang, X. Aiming at cancer in vivo: ferroptosis-inducer delivered by nanoparticles. Cell Chem. Biol. 26, 621-622 (2019).

122. Zhang, Y. et al. Imidazole ketone erastin induces ferroptosis and slows tumor growth in a mouse lymphoma model. Cell Chem. Biol. 26, 623-633.e9 (2019).

123. Zou, Y. et al. A GPX4-dependent cancer cell state underlies the clear-cell morphology and confers sensitivity to ferroptosis. Nat. Commun. 10, 1617 (2019).

124. Muendlein, H. I. et al. cFLIPL protects macrophages from LPS-induced pyroptosis via inhibition of complex II formation. Science 367, 1379-1384 (2020).

125. Malireddi, R. K. S. et al. Innate immune priming in the absence of TAK 1 drives RIPK1 kinase activityindependent pyroptosis, apoptosis, necroptosis, and inflammatory disease. J. Exp. Med. 217 jem.20191644 (2020)

126. Nagata, S. Apoptosis and clearance of apoptotic cells. Annu. Rev. Immunol. 36, 489-517 (2018)

127. Green, D. R., Oguin, T. H. \& Martinez, J. The clearance of dying cells: table for two. Cell Death. Differ. 23, 915-926 (2016)

128. Martinez, J. et al. Molecular characterization of LC3-associated phagocytosis reveals distinct roles for Rubicon, NOX2 and autophagy proteins. Nat. Cell Biol. 17, 893-906 (2015).

129. Martinez, J. et al. Noncanonical autophagy inhibits the autoinflammatory, lupus-like response to dying cells. Nature 533, 115-119 (2016).

130. Oboki, K. et al. IL-33 is a crucial amplifier of innate rather than acquired immunity. Proc. Natl Acad. Sci. USA 107, 18581-18586 (2010).

131. Rickard, J. A. et al. RIPK1 regulates RIPK3-MLKL driven systemic inflammation and emergency hematopoiesis. Cell 157, 1175-1188 (2014).

132. Schiering, C. et al. The alarmin IL-33 promotes regulatory T-cell function in the intestine. Nature 513 , 564-568 (2014).

133. Zhu, K. et al. Necroptosis promotes cell-autonomous activation of proinflammatory cytokine gene expression. Cell Death Dis. 9, 500 (2018)

134. Južnić, L. et al. SETDB 1 is required for intestinal epithelial differentiation and the prevention of intestinal inflammation. Gut 70, 485-498 (2021).

135. Wang, R. et al. Gut stem cell necroptosis by genome instability triggers bowel inflammation. Nature $\mathbf{5 8 0}$ 386-390 (2020).

136. Hildebrand, J. M. et al. A missense mutation in the MLKL brace region promotes lethal neonatal inflammation and hematopoietic dysfunction. Nat. Commun. 11, 3150 (2020).

137. Brough, D. \& Rothwell, N. J. Caspase-1-dependent processing of pro-interleukin-1beta is cytosolic and precedes cell death. J. Cell Sci. 120, 772-781 (2007).

138. Bergsbaken, T., Fink, S. L., den Hartigh, A. B., Loomis, W. P. \& Cookson, B. T. Coordinated host responses during pyroptosis: caspase-1-dependent 
lysosome exocytosis and inflammatory cytokine maturation. J. Immunol 187, 2748-2754 (2011).

139. Vanden Berghe, T. et al. An inactivating caspase-11 passenger mutation muddles sepsis research. Am. J. Respir. Crit. Care Med. 188, 120-121 (2013).

140. Case, C. L. et al. Caspase-11 stimulates rapid flagellin independent pyroptosis in response to Legionella pneumophila. Proc. Natl Acad. Sci. USA 110, 1851-1856 (2013)

141. Broz, P. et al. Caspase- 11 increases susceptibility to Salmonella infection in the absence of caspase-1. Nature 490, 288-291 (2012).

142. Mier, J. W. et al. Dissimilarities between purified human interleukin-1 and recombinant human interleukin-2 in the induction of fever, brain prostaglandin, and acute-phase protein synthesis. J. Biol. Response Mod. 4, 35-45 (1985)

143. Li, W. et al. Ferroptotic cell death and TLR4/Trif signaling initiate neutrophil recruitment after heart transplantation. J. Clin. Invest. 129, 2293-2304 (2019).

144. Allam, R. et al. Histones from dying renal cells aggravate kidney injury via TLR2 and TLR4 J. Am. Soc. Nephrol. 23, 1375-1388 (2012)

145. Nakazawa, D. et al. Histones and neutrophil extracellular traps enhance tubular necrosis and remote organ injury in ischemic AKI. J. Am . Soc. Nephrol. 28, 1753-1768 (2017)

146. Degterev, A. \& Linkermann, A. Generation of small molecules to interfere with regulated necrosis. Cell Mol. Life Sci. 73, 2251-2267 (2016).

147. von Massenhausen, A. et al. Phenytoin inhibits necroptosis. Cell Death Dis. 9, 359 (2018)

148. Souers, A. J. et al. ABT-199, a potent and selective BCL-2 inhibitor, achieves antitumor activity while sparing platelets. Nat. Med. 19, 202-208 (2013).

149. Llabani, E. et al. Diverse compounds from pleuromutilin lead to a thioredoxin inhibitor and inducer of ferroptosis. Nat. Chem. 11, 521-532 (2019).

150. Degterev, A. et al. Chemical inhibitor of nonapoptotic cell death with therapeutic potential for ischemic brain injury. Nat. Chem. Biol. 1, 112-119 (2005).

151. Degterev, A., Maki, J. L. \& Yuan, J. Activity and specificity of necrostatin-1, small-molecule inhibitor of RIP1 kinase. Cell Death Differ. 20, 366 (2013).

152. Dannappel, M. et al. RIPK1 maintains epithelial homeostasis by inhibiting apoptosis and necroptosis. Nature 513, 90-94 (2014).

153. Kaiser, W. J. et al. RIP1 suppresses innate immune necrotic as well as apoptotic cell death during mammalian parturition. Proc. Natl Acad. Sci. USA 111, 7753-7758 (2014)

154. Takahashi, N. et al. RIPK 1 ensures intestinal homeostasis by protecting the epithelium against apoptosis. Nature 513, 95-99 (2014).

155. Mandal, P. et al. RIP3 induces apoptosis independent of pronecrotic kinase activity. Mol. Cell 56, 481-495 (2014).

156. Pierotti, C. L. et al. Potent inhibition of Necroptosis by simultaneously targeting multiple effectors of the pathway. ACS Chem. Biol. 15, 2702-2713 (2020).

157. Del Re, D. P., Amgalan, D., Linkermann, A., Liu, Q. \& Kitsis, R. N. Fundamental mechanisms of regulated cell death and implications for heart disease. Physiol. Rev. 99, 1765-1817 (2019).

158. Fang, X. et al. Ferroptosis as a target for protection against cardiomyopathy. Proc. Natl Acad. Sci. USA 116, 2672-2680 (2019).

159. Gao, M., Monian, P., Quadri, N., Ramasamy, R. \& Jiang, X. Glutaminolysis and transferrin regulate ferroptosis. Mol. Cell 59, 298-308 (2015).

160. Alim, I. et al. Selenium drives a transcriptional adaptive program to block ferroptosis and treat stroke. Cell 177, 1262-1279.e25 (2019)

161. Kenny, E. M. et al. Ferroptosis contributes to neuronal death and functional outcome after traumatic brain injury. Crit. Care Med. 47, 410-418 (2019).

162. Adedoyin, O. et al. Heme oxygenase- 1 mitigates ferroptosis in renal proximal tubule cells. Am. J. Physiol. Ren. Physiol. 314, F702-F714 (2018)

163. Deng, F., Sharma, I., Dai, Y., Yang, M. \& Kanwar, Y. S Myo-inositol oxygenase expression profile modulates pathogenic ferroptosis in the renal proximal tubule. J. Clin. Invest. 129, 5033-5049 (2019).

164. Martin-Sanchez, D. et al. Ferroptosis, but not necroptosis, is important in nephrotoxic folic acidinduced AKI. J. Am. Soc. Nephrol. 28, 218-229 (2017).

165. Stoppe, C. et al. The protective role of macrophage migration inhibitory factor in acute kidney injury after cardiac surgery. Sci. Transl Med. 10, eaan4886 (2018).
166. Schumann-Bischoff, A. et al. Distinct morphological features of acute tubular injury in renal allografts correlate with clinical outcome. Am. J. Physiol. Ren Physiol. 315, F701-F710 (2018)

167. Skouta, R. et al. Ferrostatins inhibit oxidative lipid damage and cell death in diverse disease models. J. Am. Chem. Soc. 136, 4551-4556 (2014).

168. Devisscher, L. et al. Discovery of novel, drug-like ferroptosis inhibitors with in vivo efficacy. J. Med. Chem. 61, 10126-10140 (2018).

169. Yagoda, N. et al. RAS-RAF-MEK-dependent oxidative cell death involving voltage-dependent anion channels. Nature 447, 864-868 (2007).

170. Yatim, N., Cullen, S. \& Albert, M. L. Dying cells actively regulate adaptive immune responses. Nat. Rev. Immunol. 17, 262-275 (2017).

171. Orozco, S. L. et al. RIPK3 activation leads to cytokine synthesis that continues after loss of cell membrane integrity. Cell Rep. 28, 2275-2287.e5 (2019).

172. Blander, J. M. Regulation of the cell biology of antigen cross-presentation. Annu. Rev. Immunol. 36, 717-753 (2018)

173. Snyder, A. G. et al. Intratumoral activation of the necroptotic pathway components RIPK 1 and RIPK3 potentiates antitumor immunity. Sci. Immunol. $\mathbf{4}$ eaaw2004 (2019).

174. Van Hoecke, L. et al. Treatment with mRNA coding for the necroptosis mediator MLKL induces antitumor immunity directed against neo-epitopes. Nat Commun. 9, 3417 (2018)

175. Chervonsky, A. V. et al. The role of Fas in autoimmune diabetes. Cell 89, 17-24 (1997).

176. Kang, S. M. et al. Fas ligand expression in islets of Langerhans does not confer immune privilege and instead targets them for rapid destruction. Nat. Med. 3, 738-743 (1997).

177. Kar, S., Kambis, T. N. \& Mishra, P. K. Hydrogen sulfide-mediated regulation of cell death signaling ameliorates adverse cardiac remodeling and diabetic cardiomyopathy. Am. J. Physiol. Heart Circ. Physiol. 316, H1237-H1252 (2019).

178. Bruni, A Bornstein, S Linkermann, A \& Shapiro, A. M. J. Regulated cell death seen through the lens of islet transplantation. Cell Transplant. 27 890-901 (2018)

179. Bruni, A. et al. Ferroptosis-inducing agents compromise in vitro human islet viability and function. Cell Death Dis. 9, 595 (2018).

180. Land, W. G., Agostinis, P., Gasser, S., Garg, A. D. \& Linkermann, A. Transplantation and damageassociated molecular patterns (DAMPs). Am J. Transplant. 16, 3338-3361 (2016)

181. Bornstein, S. R. Predisposing factors for adrenal insufficiency. N. Engl. J. Med. 360, 2328-2339 (2009).

182. Husebye, E. S., Pearce, S. H., Krone, N. P. \& Kämpe, O. Adrenal insufficiency. Lancet 397, 613-629 (2021).

183. Merke, D. P. et al. Adrenomedullary dysplasia and hypofunction in patients with classic 21-hydroxylase deficiency. N. Engl. J. Med. 343, 1362-1368 (2000).

184. Hata, Y., Chiba, T., Ohtani, M., Ishizawa, S. \& Nishida, N. An autopsy case of pneumococcal Waterhouse-Friderichsen syndrome with possible functional asplenia/hyposplenia. Int. J. Clin. Exp. Pathol. 8, 7518-7525 (2015)

185. Kanczkowski, W. et al. Role of the endothelial-derived endogenous anti-inflammatory factor Del-1 in inflammation-mediated adrenal gland dysfunction. Endocrinology 154, 1181-1189 (2013).

186. Datta, R., Waheed, A., Shah, G. N. \& Sly, W. S. Signal sequence mutation in autosomal dominant form of hypoparathyroidism induces apoptosis that is corrected by a chemical chaperone. Proc. Natl Acad. Sci. USA 104, 19989-19994 (2007)

187. Vladykovskaya, E. et al. Lipid peroxidation product 4-hydroxy-trans-2-nonenal causes endothelial activation by inducing endoplasmic reticulum stress. J. Biol. Chem. 287, 11398-11409 (2012).

188. Dixon, S. J. et al. Pharmacological inhibition of cystine-glutamate exchange induces endoplasmic reticulum stress and ferroptosis. eLife 3, e02523 (2014).

189. Marx, K. et al. Cell death induced by $131 \mathrm{I}$ in a differentiated thyroid carcinoma cell line in vitro: necrosis or apoptosis? Nucl. Med. Commun. 27 353-358 (2006)

190. Liu, J. et al. Excessive iodine promotes pyroptosis of thyroid follicular epithelial cells in Hashimoto's thyroiditis through the ROS-NF-kappaB-NLRP3 pathway. Front. Endocrinol. 10, 778 (2019).

191. Kodama, T. et al. Transient thyrotoxicosis associated with infarction of a large thyroid adenoma. Endocrinol. Jpn 34, 779-784 (1987).
192. Li, H. et al. TNF-alpha is upregulated in subacute thyroiditis and stimulates expression of miR-155-5p in thyroid follicle cells. Discov. Med. 26, 67-77 (2018).

193. Salemi, S. et al. Isolated autosomal dominant growth hormone deficiency: stimulating mutant $\mathrm{GH}-1$ gene expression drives $\mathrm{GH}-1$ splice-site selection, cell proliferation, and apoptosis. Endocrinology 148 45-53 (2007).

194. Ariyasu, D., Yoshida, H., Yamada, M. \& Hasegawa, Y. Endoplasmic reticulum stress and apoptosis contribute to the pathogenesis of dominantly inherited isolated $\mathrm{GH}$ deficiency due to $\mathrm{GH} 1$ gene splice site mutations. Endocrinology 154, 3228-3239 (2013).

195. Chesnokova, V. et al. Growth hormone is permissive for neoplastic colon growth. Proc. Natl Acad. Sci. USA 113, E3250-E3259 (2016)

196. Yan, Y. et al. Aortic cell apoptosis in rat primary aldosteronism model. J. Huazhong Univ. Sci. Technolog. Med. Sci. 30, 385-390 (2010).

197. Yang, Y. et al. Primary aldosteronism: KCNJ5 mutations and adrenocortical cell growth. Hypertension 74, 809-816 (2019)

198. Duan, T. et al. Role of peroxiredoxin 2 in H2O2induced oxidative stress of primary Leydig cells Mol. Med. Rep. 13, 4807-4813 (2016).

199. Luo, Y. et al. Hypersensitivity of primordial germ cells to compromised replication-associated DNA repair involves ATM-p53-p21 signaling. PLoS Genet. 10, e1004471 (2014)

200. Kastenhuber, E. R. \& Lowe, S. W. Putting p53 in context. Cell 170, 1062-1078 (2017).

201. Tarangelo, A. et al. p53 suppresses metabolic stress-induced ferroptosis in cancer cells. Cell Rep. 22 569-575 (2018)

202. Moon, S. H. et al. p53 represses the mevalonate pathway to mediate tumor suppression. Cell 176 564-580.e19 (2019).

203. Brissot, P. et al. Haemochromatosis. Nat. Rev. Dis. Primers 4, 18016 (2018).

204. Nelson, J. E., Brunt, E. M. \& Kowdley, K. V. Lower serum hepcidin and greater parenchymal iron in nonalcoholic fatty liver disease patients with $\mathrm{C} 282 \mathrm{Y}$ HFE mutations. Hepatology 56, 1730-1740 (2012).

205. Qi, J., Kim, J. W., Zhou, Z., Lim, C. W. \& Kim, B. Ferroptosis affects the progression of nonalcoholic steatohepatitis via the modulation of lipid peroxidation-mediated cell death in mice. $\mathrm{Am}$. J. Pathol. 190, 68-81 (2020)

206. Tsurusaki, S. et al. Hepatic ferroptosis plays an important role as the trigger for initiating inflammation in nonalcoholic steatohepatitis. Cell Death Dis. 10, 449 (2019).

207. Wachenfeld, C. et al. Discerning malignancy in adrenocortical tumors: are molecular markers useful? Eur. J. Endocrinol. 145, 335-341 (2001).

208. Reincke, M. et al. p53 mutations in human adrenocortical neoplasms: immunohistochemical and molecular studies. J. Clin. Endocrinol. Metab. 78 790-794 (1994).

209. Saeger, W. et al. High diagnostic accuracy of adrenal core biopsy: results of the German and Austrian adrenal network multicenter trial in 220 consecutive patients. Hum. Pathol. 34, 180-186 (2003).

210. Giordano, T. J. et al. Molecular classification and prognostication of adrenocortical tumors by transcriptome profiling. Clin. Cancer Res. 15 , 668-676 (2009).

211. Zheng, S. et al. Comprehensive pan-genomic characterization of adrenocortical carcinoma. Cancer Cell 30, 363 (2016)

212. de Cubas, A. A. et al. DNA methylation profiling in pheochromocytoma and paraganglioma reveals diagnostic and prognostic markers. Clin. Cancer Res. 21, 3020-3030 (2015).

213. Khosla, S. et al. Loss of heterozygosity suggests multiple genetic alterations in pheochromocytomas and medullary thyroid carcinomas. J. Clin. Invest. 87 1691-1699 (1991).

214. Fankhauser, M. et al. Synergistic highly potent targeted drug combinations in different pheochromocytoma models including human tumor cultures. Endocrinology 160, 2600-2617 (2019).

215. Velasquez, G. et al. The "ring sign" of necrotic pheochromocytoma. Radiology 131, 69-71 (1979).

216. Short, I. A. \& Padfield, P. L. Malignant phaeochromocytoma with severe constipation and myocardial necrosis. Br. Med. J. 2, 793-794 (1976).

217. Delaney, J. P. \& Paritzky, A. Z. Necrosis of a pheochromocytoma with shock. N. Engl. J. Med. 280 1394-1395 (1969). 
218. Nyman, D. \& Wahlberg, P. Necrotic phaeochromocytoma with gastric haemorrhage, shock, and uncommonly high catecholamine excretion. Acta Med. Scand. 187, 381-383 (1970).

219. Carpenter, A. A. \& Kunin, A. S. Pheochromocytoma with acute tubular necrosis. Report of a case. N. Engl. J. Med. 265, 986-988 (1961).

220. Liu, C. L. et al. Targeting the pentose phosphate pathway increases reactive oxygen species and induces apoptosis in thyroid cancer cells. Mol. Cell. Endocrinol. 499, 110595 (2020).

221. Iannetti, A. et al. The neutrophil gelatinase-associated lipocalin (NGAL), a NF-kB-regulated gene, is a survival factor for thyroid neoplastic cells. Proc. Natl Acad. Sci. USA 105, 14058-14063 (2008).

222. Riegman, M., Bradbury, M. S. \& Overholtzer, M. Population dynamics in cell death: mechanisms of propagation. Trends Cancer 5, 558-568 (2019).

223. Vanden Berghe, T. et al. Determination of apoptotic and necrotic cell death in vitro and in vivo. Methods 61, 117-129 (2013)
224. Howard, S. C., Jones, D. P. \& Pui, C. H. The tumor lysis syndrome. N. Engl. J. Med. 364, 1844-1854 (2011).

\section{Acknowledgements}

A.L. is supported by a Heisenberg-Professorship granted by the German Research Foundation (DFG), project number 324141047. Work in the Linkermann Lab and the Bornstein Lab is funded by Medical Clinic 3, University Hospital Car Gustav Carus Dresden, Germany, and supported by the SFBTRR 205, SFB-TRR 127 and the international research training group (IRTG) 2251. This work was further supported by the transCampus to S.R.B and A.L. N.P.K was supported by the DFG, project KR3363/3-1. F.B. is supported by the Hochschulmedizin Zürich through the Flagship project Immuno-TarCET. We would like to thank all members of the Linkermann Lab for the ongoing discussions. We cordially thank R. Drtina for her assistance with preparing the first draft of the manuscript.

\section{Author contributions}

W.T., A.B. and A.L. researched data for the article, contributed to the discussion of the content, wrote the article, and reviewed and/or edited the article before submission. F.B., G.E., M.F. M.K., N.P.K., M.R. and S.R.B. researched data for the article, contributed to the discussion of the content, and reviewed and/or edited the article before submission.

\section{Competing interests}

The authors declare no competing interests.

\section{Peer review information}

Nature Reviews Endocrinology thanks J. Yuan and $\mathrm{N}$. Chattipakorn and the other, anonymous, reviewer(s) for their contribution to the peer review of this work.

\section{Publisher's note}

Springer Nature remains neutral with regard to jurisdictional claims in published maps and institutional affiliations.

\section{Supplementary information}

The online version contains supplementary material available at https://doi.org/10.1038/s41574-021-00499-w.

(C) Springer Nature Limited 2021 